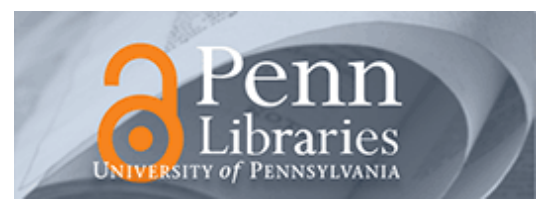

University of Pennsylvania

ScholarlyCommons

Accounting Papers

Wharton Faculty Research

$11-2015$

\title{
Delegated Trade and the Pricing of Public and Private Information
}

Daniel J. Taylor

University of Pennsylvania

Robert E. Verrecchia

University of Pennsylvania

Follow this and additional works at: https://repository.upenn.edu/accounting_papers

Part of the Accounting Commons, and the Economics Commons

\section{Recommended Citation}

Taylor, D. J., \& Verrecchia, R. E. (2015). Delegated Trade and the Pricing of Public and Private Information. Journal of Accounting and Economics, 60 (2-3), 8-32. http://dx.doi.org/10.1016/j.jacceco.2015.07.002

This paper is posted at ScholarlyCommons. https://repository.upenn.edu/accounting_papers/20

For more information, please contact repository@pobox.upenn.edu. 


\title{
Delegated Trade and the Pricing of Public and Private Information
}

\author{
Abstract \\ We extend a standard, rational expectation model of trade to incorporate the possibility of individual \\ investors delegating their trades to an informed financial intermediary. In the presence of delegated trade, \\ we show that a firm's risk premium is a function of both the firm's exposure to a common risk factor and \\ idiosyncratic characteristics of the firm's information environment. We show that even in a large \\ economy, priced risks can manifest in the form of both idiosyncratic firm characteristics and common \\ risk factors; as a consequence, factor-based asset pricing tests cannot rule out that a particular risk is \\ priced.

\section{Keywords} \\ delegated trade, institutional investors, imperfect competition, risk premium, expected returns, \\ information quality, accounting quality, idiosyncratic risk, asset-pricing tests \\ Disciplines \\ Accounting | Economics
}




\title{
Delegated Trade and the Pricing of Public and Private Information
}

\author{
Daniel J. Taylor \\ dtayl@wharton.upenn.edu \\ Robert E. Verrecchia \\ verrecchia@wharton.upenn.edu \\ The Wharton School \\ University of Pennsylvania
}

This Draft: July 7, 2015

We thank Mary Barth, Jeremy Bertomeu, Judson Caskey, Qi Chen, John Core, Paul Fischer, Mirko Heinle, Wayne Landsman, Terry Shevlin, Jerry Zimmerman (editor), two anonymous referees, Matt and Rob Bloomfield (discussants) and seminar participants at Carnegie Mellon University, London Business School, University of North Carolina (Chapel Hill), Ohio State University, Rice University, and the 2014 JAE conference for helpful comments. 


\title{
Delegated Trade and the Pricing of Public and Private Information
}

\begin{abstract}
:
We extend a standard, rational expectation model of trade to incorporate the possibility of individual investors delegating their trades to an informed financial intermediary. In the presence of delegated trade, we show that a firm's risk premium is a function of both the firm's exposure to a common risk factor and idiosyncratic characteristics of the firm's information environment. We show that even in a large economy, priced risks can manifest in the form of both idiosyncratic firm characteristics and common risk factors; as a consequence, factor-based asset pricing tests cannot rule out that a particular risk is priced.
\end{abstract}

Keywords: delegated trade; institutional investors; imperfect competition; risk premium; expected returns; information quality; accounting quality; idiosyncratic risk; asset-pricing tests

JEL Classification: G11, G12, G14, G31 


\section{Introduction}

There is an ongoing debate on whether characteristics of a firm's information environment are priced. Existing economic theory suggests that, in large economies populated by rational investors, the firm's information environment has no effect on the risk premium incremental to the firm's exposure to common risk factors, i.e., factor-betas. Based on this theory, a large empirical literature examines whether the quality of public information (e.g., "accounting quality") represents a separate, common risk factor (see Shevlin, 2013 for a review). ${ }^{1}$

A standard assumption in this literature is that individual investors (both informed and uninformed) trade on their own accounts, or "self-direct" their trades. In this paper, we extend a standard, rational expectation model of trade to incorporate the possibility that individual investors can delegate their trades to a privately informed financial intermediary: for example, an institutional investor. We show that delegation results in characteristics of the firm's information environment - specifically, the qualities of public and private information and idiosyncratic cash flow volatility - affecting the risk premium incremental to factor-betas. In our model, priced risk manifests in the form of both idiosyncratic firm characteristics and factor-betas: as a consequence, factor-based asset pricing tests cannot rule out that a particular risk is priced.

We begin our analysis by considering the standard benchmark case of a single firm, where the firm's cash flow is comprised of two components: a systematic component resulting from exposure to a common risk factor and an idiosyncratic component. We assume the economy is populated by a large number of rational, atomistic investors; each individual investor behaves as a price taker and self-directs his trades. In this setting, we show that the expression for the risk premium (the difference between a firm's expected cash flow and

\footnotetext{
${ }^{1}$ By "common risk factor" we refer to an aggregate variable that takes the same value for all firms, e.g., one of the three Fama-French factors. By "factor-beta" we refer to the firm's exposure, or covariance, with a common risk factor. By "characteristic of a firm" or "firm characteristic," we refer to an idiosyncratic attribute of the firm, e.g., a firm's "accounting quality."
} 
its expected price) effectively reduces to that of the Capital Asset Pricing Model (CAPM).

Next, we assume that an exogenous fraction of investors in the economy delegate their trades to a financial intermediary who has private information about the firm. Large economies generally result in perfect competition and, for this reason, characteristics of the firm's information environment are not thought to affect the firm's risk premium incremental to factor-betas. However, the presence of an intermediary prevents the seeming inevitability of a large economy becoming perfectly competitive. The intuition for this result is that, as the economy grows, the intermediary's client base (e.g., "assets under management") grows in tandem, and thus even though any single investor's effect on price declines as the economy gets larger, the intermediary's effect on price does not. As the economy grows, any (potential) decline in the intermediary's effect on price is offset by a corresponding increase in the intermediary's larger aggregate demand order that results from trading on behalf of more clients. The ongoing presence of this market imperfection results in imperfect competition being sustained despite the fact that the economy is large. Because the economy continues to be imperfectly competitive, the qualities of public and private information and the firm's idiosyncratic cash flow volatility affect the risk premium incremental to the factor-betas.

Finally, we extend the model to allow the following elements to be endogenous: (i) the fraction of investors who delegate their trades; (ii) the number of intermediaries; and (iii) the fee that intermediaries charge their clients. Here, one can think of our analysis as extending Grossman and Stiglitz (1980) to include features from Kyle (1989). Rather than have investors pay a fixed fee to acquire private information (as in Grossman and Stiglitz, 1980), we assume that investors pay a fixed fee to delegate their trading decisions to a privately informed financial intermediary, where the fee is endogenously determined by competition among the intermediaries.

One way to motivate this modeling approach is to appeal to the notion that the costs of acquiring private information are so large that no single, individual investor could bear the 
entirety of the costs. Instead, the chief role of the intermediary is to acquire private information, trade on behalf of his clients, and then charge each individual client a very small fee as compensation for his services. The collection of small fees reimburses the intermediary for large information acquisition costs. Given the significant presence of institutional investors in large economies, we believe our analysis of delegated trade offers a more compelling story about how information is gathered and disseminated in a large economy with atomistic investors. ${ }^{2}$

Our analysis integrates the economics literature on delegated trade with the accounting literature on the effect of accounting information on asset prices. Several studies in the economics literature examine the implications of investors delegating their investment decisions to a financial intermediary. Broadly stated, this literature attempts to understand the efficiency of such arrangements (e.g., Admati and Pfleiderer, 1990; Garcia and Vanden, 2009; Kyle et al., 2011) and how the incentives of portfolio managers affect equilibrium prices (see Cuoco and Kaniel, 2011, for a review). However, this literature does not distinguish between different sources of priced risk: factor-betas or characteristics of the information environment.

There are many studies in the accounting literature that examine the pricing of public and private information, but do so from the perspective that investors' trades are self-directed. Self-directed trade among atomistic investors in large economies typically results in perfect competition, and perfectly competitive markets typically have the feature that a firm's information environment does not affect the premium incremental to the firm's factor-beta (e.g., Hughes et al., 2007; Lambert et al., 2007; Gao, 2010). ${ }^{3}$ For example, Lambert et al. (2007)

\footnotetext{
${ }^{2}$ French (2008) suggests that the fraction of common equity in the U.S. indirectly held by individual investors through-open ended mutual funds increased from $4.6 \%$ in 1980 to $32.4 \%$ in 2007, whereas the fraction of common equity directly held by individual investors declined from $47.9 \%$ to $21.5 \%$ over the same period.

${ }^{3}$ Chen et al. (2013) and Clinch (2013) study similar settings, but in the presence of departures from rationality (i.e. non-Bayesian behavior).
} 
posit a model of perfect competition where the quality of public information affects expected returns exclusively through factor-loadings. In contrast, Lambert et al. (2012) study the pricing of information in a setting where the market is imperfectly competitive as a consequence of some investors anticipating the effect of their demand on price (see also Caskey et al., 2014; Lambert and Verrecchia, 2014). However, as these investors become small in relation to the size of the economy, the anticipated effect vanishes, the market becomes perfectly competitive, and public and private information do not affect the risk premium incremental to factor-betas. Relative to this literature (see Bertomeu and Cheynel, 2014, for a review), the distinguishing feature of our analysis is that we consider the possibility of delegated trade. Delegated trade inhibits a large economy from becoming perfectly competitive; as a consequence, characteristics of the firm's information environment manifest in asset prices incremental to the firm's factor-beta despite the economy being large.

It is easy to imagine a circumstance where the risk premium is characterized exclusively in terms of factor-betas (e.g., the CAPM). However, given the mounting empirical evidence that firm characteristics affect asset prices, the purpose of this paper is to identify and examine a circumstance in which characteristics of the firm's information environment are priced incremental to factor-betas, despite the economy being large. The insights from our model have several implications for empirical work, which we discuss in detail in Section 6. First, to the extent that a particular firm characteristic is related to the quality of public information, the quality of private information, and/or the firm's idiosyncratic cash flow volatility, our analysis suggests that the characteristic will be priced incremental to factor-betas. Second, because priced risks can manifest in the form of both idiosyncratic firm characteristics and factor-betas, our results suggest that factor-based asset pricing tests cannot rule out that a particular risk is priced - only that it is priced as a common risk factor. Third, our results suggest considerable variation in the effect of public information on illiquidity and expected returns. For example, our analysis suggests the effect of public information on illiquidity 
and expected returns varies with the quality of private information, with systematic cash flow volatility, and with the costs borne by the intermediary. Finally, our analysis provides insight on how the information environment influences individual investors' decisions to delegate their trades.

The remainder of the paper proceeds as follows. Section 2 provides an overview of our analysis. Section 3 considers the role of delegated trade assuming an exogenous fraction of investors delegate trades to a single information intermediary. Section 4 extends the model to a setting where the following elements are endogenous: the fraction of investors who delegate trades; the number of intermediaries; and the fee that intermediaries charge to their clients. Section 5 extends the model to a setting where investors can trade the common factor. Section 6 discusses the implications of our model for empirical work. Section 7 concludes.

\section{Overview}

\subsection{Benchmark case}

In this section, we discuss the setup of our model and our assumptions. We do this in the context of the standard benchmark case of a single risky asset (e.g., a firm). To start, we solve for the risk premium in the absence of delegated trade, and show that the expression for the risk premium effectively reduces to that of the CAPM.

Trading takes place in a one-period capital market comprised of $N$ rational investors, each with negative exponential utility with constant absolute risk tolerance $\tau$. Investors can trade shares of a riskless bond, whose return and price we normalize to 0 and 1, respectively, and shares of a risky asset. Shares of the risky asset are traded at a market clearing price $\tilde{P}_{a}$ and shareholders realize an uncertain terminal cash flow of $\tilde{V}_{a}$ after trade occurs, where henceforth we use a tilde, i.e., , to denote a random variable. The risk premium investors 
demand to hold shares of the risky asset is defined as the difference between the expected cash flow and expected price, $E\left[\tilde{V}_{a}-\tilde{P}_{a}\right]$.

We assume the risky asset's cash flow is comprised of two components: a systematic component resulting from exposure to a common risk factor and an idiosyncratic component. We represent the risky asset's cash flow by

$$
\tilde{V}_{a}=\mu_{a}+\beta \cdot \tilde{F}+\tilde{\varepsilon}
$$

where $\mu_{a}$ and $\beta$ are fixed parameters, $\tilde{F}$ has a normal distribution with mean 0 and variance $\sigma_{F}^{2}$, and $\tilde{\varepsilon}$ has a normal distribution with mean 0 and variance $\sigma_{\varepsilon}^{2}$ and is independent of $\tilde{F}$. ${ }^{4}$ We refer to $\beta$ as the risky asset's "factor-beta," $\sigma_{F}^{2}$ as "systematic cash flow volatility," and $\sigma_{\varepsilon}^{2}$ as "idiosyncratic cash flow volatility." As is standard in the literature, $\sigma_{\varepsilon}^{2}$ represents investors' assessment of idiosyncratic cash flow volatility. Investors' assessment of idiosyncratic cash flow volatility is driven by the sum of two (idiosyncratic) forces: the real volatility of cash flow and common knowledge about that cash flow. This implies that either a reduction in real volatility or an increase in the quality of public information about volatility works to decrease $\sigma_{\varepsilon}^{2}$.

Consider the standard benchmark case where investors have homogenous beliefs and the market is perfectly competitive (i.e., there is no private information and all investors are price takers). It is straightforward to show that the expression for the risk premium reduces to

$$
E\left[\tilde{V}_{a}-\tilde{P}_{a}\right]=\frac{1}{N \tau}\left(\beta^{2} \sigma_{F}^{2}+\sigma_{\varepsilon}^{2}\right)
$$

In effect, the risky asset is priced based on its expected cash flow less a discount for the volatility of the risky asset's cash flow and the economy's aggregate risk tolerance (i.e.,

\footnotetext{
4 Without loss of generality, we assume cash flow is generated by exposure to a single risk factor. The results extend to a multifactor specification of cash flow with multiple factor-betas.
} 
$N \tau)$. This equation makes clear that the risk premium will vanish as $N$ becomes large (i.e., $N \rightarrow \infty)$ and the risky asset will be priced at the expected value of terminal cash flow. The intuition for this result is that the economy's aggregate risk tolerance gets progressively larger as the economy grows, while total risk stays fixed. In other words, in a large economy, the only risk that is meaningful is the risk that grows with the economy. This theme pervades the literature.

Consider the classic CAPM. Although the CAPM is couched in terms of returns and not cash flows, using a standard technique along the lines of Fama (1976) one can convert the CAPM from a model of returns to a model of cash flows. In the CAPM, in order for "systematic risk" to be meaningful, the risk associated with the systematic component of cash flow must grow in proportion to the number of investors: in other words, it must be the case that $\sigma_{F}^{2}=N \sigma_{f}^{2}$, where $\sigma_{f}^{2}$ represents the level of risk per capita (or per investor). The chief takeaway from the CAPM is that only the risk associated with the systematic portion of cash flow grows with the economy, and thus only this portion is priced in a large economy. Risk associated with the idiosyncratic portion of cash flow does not grow with the economy, and thus its effect on price vanishes in a large economy.

Within the context of the above example and similar to the CAPM, suppose the risk associated with the common factor grows in proportion to the number of investors in the economy (i.e., $\sigma_{F}^{2}=N \sigma_{f}^{2}$ ). Then the expression for the risk premium reduces to

$$
E\left[\tilde{V}_{a}-\tilde{P}_{a}\right]=\frac{1}{\tau} \beta^{2} \sigma_{f}^{2}+\frac{1}{N \tau} \sigma_{\varepsilon}^{2}
$$

Here, risk associated with the common factor grows with the economy whereas risk associated with the idiosyncratic portion does not. As such, as $N$ becomes large, the risk premium reduces to $\tau^{-1} \beta^{2} \sigma_{f}^{2}$ and depends exclusively on the systematic portion. Note that in this case the expression for the risk premium is effectively that of the CAPM. This simple benchmark 
case provides the intuition for why, in economies comprised by a large number of investors, the risk premium is only thought to be a function of factor-betas.

Henceforth, as in the CAPM, we assume that risk associated with the common factor grows in proportion to the number of investors in the economy, and the risk associated with the idiosyncratic portion of cash flow does not.

\subsection{Delegated trade}

Now we posit the existence of an exogenous fraction $\alpha \in[0,1]$ of investors in the economy who delegate their trading decisions to a single financial intermediary. In Section 4 we allow both italicized features to be endogenous.

The intermediary has private information about the idiosyncratic component of the risky asset's cash flow, $\tilde{\varepsilon}$. We represent the private information by the statistic $\tilde{y}$, where $\tilde{y}$ is

defined by $\tilde{y}=\tilde{\varepsilon}+\tilde{\xi}$, where $\tilde{\xi}$ has a normal distribution with mean 0 and variance $\sigma_{\xi}^{2}$ (and is independent of all other variables): $\tilde{\xi}$ represents the error or noise in the intermediary's private knowledge about $\tilde{\varepsilon}$. The intermediary does not share his private information with his $\alpha N$ clients, but rather trades on their behalf based on his private information. We assume the intermediary trades to maximize each client's expected utility (conditional on his private information) and treats each client identically (because all investors are identical ex ante). Formally, the intermediary executes a single trade $\alpha N \cdot D_{I a}$ on behalf of his clients, where $D_{I a}$ maximizes each client's expected utility conditional on the intermediary's private information, $\tilde{y}$, and the fact that the intermediary's aggregate demand order is $\alpha N \cdot D_{I a}$.

The execution of a single trade $\alpha N D_{I a}$ implies that in maximizing his clients' expected utility, the intermediary must take into consideration the impact his clients' aggregate demand has on the price at which their trades are executed. This, in turn, results in market competition being imperfect. Because $\alpha N D_{I a}$ grows with the size of the economy (through 
$N$ ), the market remains imperfect regardless of size. This market imperfection results in the risk premium being a function of the product of Kyle's $\lambda$ (which measures the extent to which a unit of demand affects the price at which trades are executed) and $\alpha N D_{I a}$ (aggregate demand), where $\lambda$ and $\alpha N D_{I a}$ are endogenous variables that depend on both systematic and idiosyncratic cash flow volatility. Because the product $\lambda \cdot \alpha N D_{I a}$ does not vanish as the economy becomes large, the risk premium depends on systematic and idiosyncratic risks. Alternatively, when market competition is perfect, investors presume that their demands have no effect on price (which is equivalent to investors presuming that $\lambda=0$ ) and this presumption must hold in equilibrium. Thus, under perfect competition the effect of $\lambda \cdot \alpha N D_{I a}$ on the risk premium is 0 , and hence risks associated with systematic and idiosyncratic cash flows remain additive and separable, such that the latter vanishes as $N$ increases.

For example, if - in the absence of an intermediary - each of $\alpha N$ investors directly observed $\tilde{y}$ and competed against one another, as well as against each of the $(1-\alpha) N$ uninformed investors, then the analysis would reduce to the seminal discussion in Grossman and Stiglitz (1980) where percentages of informed and uninformed investors compete as price takers (i.e., perfect competition). As in eqn. (2), the resulting risk premium would preserve the additive and separable nature of the risks associated with systematic and idiosyncratic cash flows such that the latter would vanish as $N$ became large.

\section{Capital market with delegated trade}

\subsection{Model setup}

To review the assumptions to this point, we consider a one-period capital market comprised of $N$ identical (rational) investors, each with negative exponential utility and constant absolute

risk tolerance $\tau$. We assume that the risky asset has an (uncertain) cash flow $\tilde{V}_{a}$ represented 
by $\tilde{V}_{a}=\mu_{a}+\beta \tilde{F}+\tilde{\varepsilon}$, where $\tilde{F}$ and $\tilde{\varepsilon}$ have independent normal distributions with mean 0 and variances $\sigma_{F}^{2}=N \sigma_{f}^{2}$ and $\sigma_{\varepsilon}^{2}$, respectively. We incorporate private information into our model by assuming that a fraction $\alpha \in(0,1)$, of the investors in the economy delegate their trading decisions to an intermediary who has private information about the risky asset's idiosyncratic component, $\tilde{\varepsilon}$. The private information is represented by the statistic $\tilde{y}$, where $\tilde{y}$ is defined by $\tilde{y}=\tilde{\varepsilon}+\tilde{\xi}$, where $\tilde{\xi}$ has a normal distribution with mean 0 and variance $\sigma_{\xi}^{2}$ (and is independent of all other variables). Henceforth we let $\pi_{\varepsilon}=\frac{1}{\sigma_{\varepsilon}^{2}}$ and $\pi_{\xi}=\frac{1}{\sigma_{\xi}^{2}}$ represent the reciprocals of the variances of $\tilde{\varepsilon}$ and $\tilde{\xi}$, respectively: as such, " $\pi$ " denotes the precision of a random variable. Appendix A contains a table of notation used in the paper.

Conditional on $\tilde{y}=y$, investors assess the expected cash flow of the risky asset to be $E\left[\tilde{V}_{a} \mid y\right]=\mu_{a}+\frac{\pi_{\xi}}{\pi_{\varepsilon}+\pi_{\xi}} y$ with variance

$$
\operatorname{Var}\left[\tilde{V}_{a} \mid y\right]=\beta^{2} \operatorname{Var}[\tilde{F}]+\operatorname{Var}[\tilde{\varepsilon} \mid y]=N \beta^{2} \sigma_{f}^{2}+\left(\pi_{\varepsilon}+\pi_{\xi}\right)^{-1}
$$

Let $D_{t a}$ represent an investor's demand for a percentage of the risky asset's cash flow, where $t \in[I, U]$ represents an investor's type: $t=I$ if the investor is a client of the intermediary and is thus "informed," or $t=U$ if the investor is not a client of the intermediary and is thus "uninformed." In keeping with the noisy rational expectations literature, we use the naming conventions "informed" and "uninformed," and refer to clients of the intermediary as "informed" because their trades (while made by the intermediary) are based on private information. Because informed investors' demand contains private information, there has to be noise to preclude the possibility of uninformed investors inferring this information perfectly by conditioning their beliefs on the price of the asset. ${ }^{5} \mathrm{~A}$ standard technique for adding noise in the (noisy) rational expectations literature is to assume that the supply of the risky asset available for trade among informed and uninformed investors is uncertain.

\footnotetext{
${ }^{5}$ While in principle there is nothing wrong with uninformed investors making perfect inferences, the resulting equilibrium is thought to be uninteresting.
} 
We add uncertainty by assuming that $\alpha N \cdot D_{I a}+(1-\alpha) N \cdot D_{U a}=\tilde{x}$, where $\tilde{x}$ has a normal distribution with mean 1 and precision $\pi_{x}$ (and is independent of all other variables). ${ }^{6}$

\subsection{Informed investors' demand}

There are four key steps in our analysis. First, we determine informed investors' demand for the risky asset. Second, we determine uninformed investors' demand. Third, we determine the risky asset's market-clearing price. Finally, we analyze the risky asset's risk premium.

Recall that the intermediary treats all clients identically, and determines each client's demand such that $D_{I a}$ maximizes the client's expected utility conditional on the intermediary's private information, $\tilde{y}$. In coordinating trade, the intermediary conjectures the following association among the price of the risky asset, $P_{a}$, the aggregate demand of informed investors, $\alpha N \cdot D_{I a}$, and the realization of the supply of the risky asset available for trade, $\tilde{x}=x$ :

$$
P_{a}=\mu_{a}+\theta+\lambda\left(\alpha N \cdot D_{I a}-x\right)
$$

where $\theta$ and $\lambda$ are endogenous variables whose values need to be determined to ensure that this conjecture is sustained in equilibrium. ${ }^{7}$ Here, $\lambda>0$ captures the extent to which the aggregate demand of the informed investors, $\alpha N \cdot D_{I a}$, affects the price at which trades are executed: as $\lambda$ increases (decreases), trade in the risky asset becomes more (less) illiquid. While the realization of $\tilde{x}$ is unknown, when price is in the form of eqn. (4) the intermediary can infer the realization $\tilde{x}=x$ by conditioning his beliefs on $P_{a}$ in conjunction with choosing

\footnotetext{
${ }^{6}$ Note that we express investors' demands as a percentage of the risky asset's cash flow, and then, by virtue of assuming that the mean of $\tilde{x}$ is 1 , assume that $100 \%$ of the cash flow is available for trade on average: this assumption is without loss of generality.

7 A belief implicit in the intermediary's conjecture about the behavior of price is that random supply, $\tilde{x}$, acts like a large, uninformed block trade; in other words, like a large, uninformed investor who, despite being uninformed, nonetheless makes markets illiquid through the impact of his large demand on price. As such - and as in the case of a block trade - the market will be illiquid (through $x$ ) even in the absence of informed trade (i.e., $\alpha=0$ ): see the discussion in Appendix C.
} 
$D_{I a}{ }^{8}$ Thus, without loss of generality we assume that the intermediary "knows" $x$.

Conditional on $\tilde{y}=y$ and $\tilde{x}=x$, the intermediary determines the $D_{I a}$ that maximizes a client's expected utility by solving the following objective function:

$$
\begin{aligned}
& \max _{D_{I a}} E\left[-\exp \left[-\frac{1}{\tau} D_{I a}\left(\tilde{V}_{a}-P_{a}\right)\right] \mid y, x\right] \\
= & \max _{D_{I a}} E\left[-\exp \left[-\frac{1}{\tau} D_{I a}\left(\tilde{V}_{a}-\mu_{a}-\theta-\lambda\left(\alpha N D_{I a}-x\right)\right)\right] \mid y, x\right] .
\end{aligned}
$$

Using standard techniques for solving the moment-generating function of a normal distribution, this objective function can be re-expressed as:

$$
\left.\max _{D_{I a}}\left\{-\exp \left[-D_{I a} \frac{1}{\tau}\left(E[\tilde{\varepsilon} \mid y]-\theta-\lambda\left(\alpha N D_{I a}-x\right)\right)+\frac{D_{I a}^{2}}{2 \tau^{2}} \operatorname{Var}\left[\tilde{V}_{a} \mid y\right]\right]\right)\right\}
$$

The $D_{I a}$ that maximizes this objective function is given by

$$
D_{I a}=\frac{E[\tilde{\varepsilon} \mid y]-\theta+\lambda x}{2 \alpha N \lambda+\tau^{-1} \operatorname{Var}\left[\tilde{V}_{a} \mid y\right]},
$$

where $E[\tilde{\varepsilon} \mid y]=\frac{\pi_{\xi}}{\pi_{\varepsilon}+\pi_{\xi}} y$ and $\operatorname{Var}\left[\tilde{V}_{a} \mid y\right]$ is defined as in eqn. (3).

\subsection{Uninformed investors' demand}

In this subsection we determine an uninformed investor's demand for the risky asset. To start, each uninformed investor solves an objective function that is similar to the objective function of an informed investor, with two important distinctions. First, there are $(1-\alpha) N$ uninformed investors who compete with other investors to determine their demand orders. Hence, as $N$ becomes large, each uninformed investor acts as if his trade has no effect on

\footnotetext{
${ }^{8}$ For example, by conditioning his beliefs on price in conjunction with choosing $D_{I a}$ the informed investor can compute the statistic $\tilde{\phi}=\lambda^{-1}\left(\mu_{a}+\theta+\lambda \alpha N D_{I a}-\tilde{P}_{a}\right)$, and this reveals $x$.
} 
the price at which the trade will be executed, and thus behaves as a price taker. Second, an uninformed investor does not observe $\tilde{y}$. Nonetheless, as is standard in the rational expectations literature, an uninformed investor infers $\tilde{y}$ with noise by conditioning his beliefs on the price of the risky asset; through this device, he gleans (with noise) the private information that motivates informed investors' trades.

With regard to the latter, define $\gamma$ as $\gamma=\frac{\alpha N \frac{\pi_{\xi}}{\pi_{\varepsilon}+\pi_{\xi}}}{2 \alpha N \lambda+\tau^{-1} \operatorname{Var}\left[\tilde{V}_{a} \mid y\right]}$. When the price of the risky asset is of the form $P_{a}=\mu_{a}+\theta+\lambda\left(\alpha N D_{I a}-x\right)$, an uninformed investor can partially infer $\tilde{y}$ by conditioning his beliefs on $P_{a}$ (and correctly anticipating $\theta$ and $\lambda$ ): specifically, an uninformed investor computes the statistic $\tilde{q}$, where

$$
\tilde{q}=\frac{1}{\gamma} \frac{\tilde{P}_{a}-\mu_{a}-\theta}{\lambda}=\frac{1}{\gamma}\left(\alpha N \tilde{D}_{I a}-\tilde{x}\right)=\tilde{y}-\frac{\theta+\left(\lambda+\frac{1}{\alpha \tau N} \operatorname{Var}\left[\tilde{V}_{a} \mid y\right]\right) \tilde{x}}{\frac{\pi_{\xi}}{\pi_{\varepsilon}+\pi_{\xi}}}
$$

In effect, eqn. (8) allows an uninformed investor to infer $\tilde{\varepsilon}$ with error or noise:

$$
\tilde{q}=\tilde{\varepsilon}+\tilde{\xi}-\frac{\theta+\left(\lambda+\frac{1}{\alpha \tau N} \operatorname{Var}\left[\tilde{V}_{a} \mid y\right]\right) \tilde{x}}{\frac{\pi_{\xi}}{\pi_{\varepsilon}+\pi_{\xi}}}
$$

Recall that $E[\tilde{x}]=1$ and $\operatorname{Var}[\tilde{x}]=\pi_{x}^{-1}$. Eqn. (9) implies that the statistic $\tilde{q}$ has a normal distribution with mean

$$
E[\tilde{q}]=-\frac{\pi_{\varepsilon}+\pi_{\xi}}{\pi_{\xi}}\left(\theta+\lambda+\frac{1}{\alpha \tau N} \operatorname{Var}\left[\tilde{V}_{a} \mid y\right]\right)
$$

and variance $\pi_{\varepsilon}^{-1}+\pi_{\delta}^{-1}$, where

$$
\pi_{\delta}=\left(\pi_{\xi}^{-1}+\left(\frac{\lambda+\frac{1}{\alpha \tau N} \operatorname{Var}\left[\tilde{V}_{a} \mid y\right]}{\frac{\pi_{\xi}}{\pi_{\varepsilon}+\pi_{\xi}}}\right)^{2} \pi_{x}^{-1}\right)^{-1}
$$


An uninformed investor computes $\tilde{q}$ to assess $\tilde{\varepsilon}$, which in turn is used to assess $\tilde{V}_{a}$. Specifically, using standard Bayesian statistics, an uninformed investor assesses the expected cash flow of the risky asset conditional on $\tilde{q}=q$ to be

$$
E\left[\tilde{V}_{a} \mid q\right]=\mu_{a}+E[\tilde{\varepsilon} \mid q]=\mu_{a}+\frac{\pi_{\delta}}{\pi_{\varepsilon}+\pi_{\delta}}(q-E[\tilde{q}])
$$

and associates with this assessment a conditional variance

$$
\operatorname{Var}\left[\tilde{V}_{a} \mid q\right]=N \beta^{2} \sigma_{f}^{2}+\left(\pi_{\varepsilon}+\pi_{\delta}\right)^{-1}
$$

Conditional on $\tilde{q}=q$, an uninformed investor solves the following objective function

$$
\max _{D_{U a}} E\left[-\exp \left[-\frac{1}{\tau} D_{U a}\left(\tilde{V}_{a}-P_{a}\right)\right] \mid q\right]
$$

Using standard techniques for solving the moment-generating function of a normal distribution, an uninformed investor's objective function can be re-expressed as

$$
\max _{D_{U a}}\left\{-\exp \left[-D_{U a} \frac{1}{\tau}\left(E\left[\tilde{V}_{a} \mid q\right]-P_{a}\right)+\frac{D_{U a}^{2}}{2 \tau^{2}} \operatorname{Var}\left[\tilde{V}_{a} \mid q\right]\right]\right\} .
$$

The $D_{U a}$ that maximizes this objective function is given by

$$
D_{U a}=\tau \frac{E\left[\tilde{V}_{a} \mid q\right]-P_{a}}{\operatorname{Var}\left[\tilde{V}_{a} \mid q\right]}
$$

where $E\left[\tilde{V}_{a} \mid q\right]$ and $\operatorname{Var}\left[\tilde{V}_{a} \mid q\right]$ are defined as in eqns. (12) and (13). 


\subsection{An equilibrium $\lambda$}

In this subsection we determine the $\lambda$ that forms the basis for an equilibrium in our economy. Market clearing requires that the supply of the risky asset available for trade among informed and uninformed investors equals the demand, or

$$
x=\alpha N \cdot D_{I a}+(1-\alpha) N \cdot D_{U a}=\alpha N D_{I a}+(1-\alpha) N \tau \frac{\mu_{a}+\frac{\pi_{\delta}}{\pi_{\varepsilon}+\pi_{\delta}}(q-E[\tilde{q}])-P_{a}}{\operatorname{Var}\left[\tilde{V}_{a} \mid q\right]} .
$$

Recall that $\tilde{q}=\frac{1}{\gamma}\left(\alpha N \tilde{D}_{I a}-\tilde{x}\right)$. Re-arranging terms in eqn. (17) yields

$$
\begin{aligned}
P_{a}-\mu_{a}= & \frac{\operatorname{Var}\left[\tilde{V}_{a} \mid q\right]}{(1-\alpha) N \tau}\left(\alpha N D_{I a}-x\right)+\frac{\pi_{\delta}}{\pi_{\varepsilon}+\pi_{\delta}}(q-E[\tilde{q}]) \\
= & \frac{\operatorname{Var}\left[\tilde{V}_{a} \mid q\right]}{(1-\alpha) N \tau}\left(\alpha N D_{I a}-x\right)+\frac{\pi_{\delta}}{\pi_{\varepsilon}+\pi_{\delta}} \frac{1}{\gamma}\left(\alpha N D_{I a}-x\right)-\frac{\pi_{\delta}}{\pi_{\varepsilon}+\pi_{\delta}} E[\tilde{q}] \\
= & \left(\frac{\operatorname{Var}\left[\tilde{V}_{a} \mid q\right]}{(1-\alpha) N \tau}+\frac{2 \lambda+\frac{1}{\alpha \tau N} \operatorname{Var}\left[\tilde{V}_{a} \mid y\right]}{\frac{\pi_{\varepsilon}+\pi_{\delta}}{\pi_{\delta}} \frac{\pi_{\xi}}{\pi_{\varepsilon}+\pi_{\xi}}}\right)\left(\alpha N D_{I a}-x\right)-\frac{\pi_{\delta}}{\pi_{\varepsilon}+\pi_{\delta}} E[\tilde{q}] .
\end{aligned}
$$

Recall that the intermediary conjectures that price is of the form $P_{a}=\mu_{a}+\theta+\lambda\left(\alpha N D_{I a}-x\right)$. For this conjecture to be sustained, in eqn. (18) it must be the case that

$$
\lambda=\frac{\operatorname{Var}\left[\tilde{V}_{a} \mid q\right]}{(1-\alpha) N \tau}+\frac{\pi_{\delta}\left(\pi_{\varepsilon}+\pi_{\xi}\right)}{\pi_{\xi}\left(\pi_{\varepsilon}+\pi_{\delta}\right)}\left(2 \lambda+\frac{1}{\alpha \tau N} \operatorname{Var}\left[\tilde{V}_{a} \mid y\right]\right) .
$$

Because $\lambda$ appears on both sides of eqn. (19) (both directly and indirectly because $\pi_{\delta}$ is a function of $\lambda)$, define $\Lambda\left(\lambda, \tau, \pi_{\varepsilon}, \pi_{\xi}, \sigma_{f}, \pi_{x}, \alpha, \beta, N\right)$ as

$$
\Lambda(\lambda, \cdot)=\lambda-\frac{\operatorname{Var}\left[\tilde{V}_{a} \mid q\right]}{(1-\alpha) N \tau}-\frac{\pi_{\delta}\left(\pi_{\varepsilon}+\pi_{\xi}\right)}{\pi_{\xi}\left(\pi_{\varepsilon}+\pi_{\delta}\right)}\left(2 \lambda+\frac{1}{\alpha \tau N} \operatorname{Var}\left[\tilde{V}_{a} \mid y\right]\right) .
$$


To determine an equilibrium $\lambda^{*}$, say, substitute the expression for $\pi_{\delta}$ in eqn. (11) into eqn. (20) and then solve for $\Lambda\left(\lambda^{*}, \cdot\right)=0$. Having determined $\lambda^{*}$, one can solve for: $\pi_{\delta}$ in eqn. (11); and $\theta$ by noting that eqn. (18) requires $\theta=-\frac{\pi_{\delta}}{\pi_{\varepsilon}+\pi_{\delta}} E[\tilde{q}]$, which implies from eqn. (10) that

$$
\theta=\frac{\pi_{\delta}\left(\pi_{\xi}+\pi_{\varepsilon}\right)}{\pi_{\varepsilon}\left(\pi_{\xi}-\pi_{\delta}\right)}\left(\lambda+\frac{1}{\alpha \tau N} \operatorname{Var}\left[\tilde{V}_{a} \mid y\right]\right)
$$

In short, an equilibrium in our economy is characterized by a (positive) $\lambda^{*}$ that solves $\Lambda\left(\lambda^{*}, \cdot\right)=0$, and the solutions to $\pi_{\delta}$ in eqn. (11) and $\theta$ in eqn. (21).

In Appendix B we prove the following proposition.

Proposition 1. There exists a unique, positive $\lambda^{*}$ that solves $\Lambda\left(\lambda^{*}, \cdot\right)=0$ where $\Lambda(\lambda, \cdot)$ is specified as in eqn. (20). In turn, the $\lambda^{*}$ can be used to solve for a (unique) $\pi_{\delta}$ in eqn. (11) and (unique) $\theta$ in eqn. (21).

\subsection{The risk premium}

Recall that the price of the risky asset is $P_{a}=\mu_{a}+\theta+\lambda\left(\alpha N D_{I a}-x\right)$. This expression represents the risky asset's realized price: that is, the end-of-period price based on the realizations of the random variables $\tilde{y}=y, \tilde{x}=x$, and $\tilde{D}_{I a}=D_{I a}$. Because $\lambda, \theta$, and $D_{I a}$ are functions of $\pi_{\varepsilon}$ and $\pi_{\xi}$, realized price clearly depends on $\pi_{\varepsilon}$ and $\pi_{\xi}$. This does not imply, however, that the risky asset's risk premium depends on $\pi_{\varepsilon}$ and $\pi_{\xi}$. For example, let $\Delta\left(\lambda, \tau, \pi_{\varepsilon}, \pi_{\xi}, \sigma_{f}, \pi_{x}, \alpha, \beta, N\right)$ represent the risky asset's risk premium: $\Delta(\lambda, \cdot)$ is defined by

$$
\Delta(\lambda, \cdot)=E\left[\tilde{V}_{a}-\tilde{P}_{a}\right]=-\theta-\lambda E\left[\alpha N \cdot \tilde{D}_{I a}-\tilde{x}\right] \cdot 9
$$

\footnotetext{
9 As will become clear below, it is useful to define $\Delta(\lambda, \cdot)$ in eqn. (22) in terms of the 8 exogenous parameters that specify our economy (i.e., $\left.\tau, \pi_{\varepsilon}, \pi_{\xi}, \sigma_{f}, \pi_{x}, \alpha, \beta, N\right)$ and the variable $\lambda$, despite the fact that $\lambda$ is endogenous and thus is also a function of the exogenous parameters.
} 
While realized price depends on on $\pi_{\varepsilon}$ and $\pi_{\xi}$, it is a straightforward exercise to show that when all risks are finite (i.e., do not grow with the economy) the risk premium is 0 as $N$ becomes large; this result is consistent with the conventional wisdom that finite risks vanish when the number of investors becomes large.

However, when risk associated with the common factor grows with the economy, $\Delta(\lambda, \cdot)$ is a function of $\pi_{\varepsilon}$ and $\pi_{\xi}$. Specifically, when $\sigma_{F}^{2}=N \sigma_{f}^{2}$ and $N$ is large, $\Delta\left(\lambda^{*}, \cdot\right)$ reduces to

$$
\Delta\left(\lambda^{*}, \cdot\right)=\frac{\left(\alpha \lambda^{*}+\tau^{-1} \beta^{2} \sigma_{f}^{2}\right) \lambda^{*}-\frac{\alpha \pi_{\xi} \pi_{x}}{\pi_{\varepsilon}\left(\pi_{\varepsilon}+\pi_{\xi}\right)}}{2 \alpha \lambda^{*}+\tau^{-1} \beta^{2} \sigma_{f}^{2}}
$$

here $\Delta\left(\lambda^{*}, \cdot\right)$ is positive and depends on $\pi_{\varepsilon}$ and $\pi_{\xi}$, both directly and indirectly through $\lambda^{*}$ (because $\lambda^{*}$ solves $\Lambda\left(\lambda^{*}, \cdot\right)=0$, and $\Lambda(\lambda, \cdot)$ is a function of $\pi_{\varepsilon}$ and $\left.\pi_{\xi}\right)$. In Appendix B we prove the following proposition.

Proposition 2. The asset's risk premium is positive and depends on $\pi_{\varepsilon}$ and $\pi_{\xi}$.

In Appendix B we prove the following two corollaries to Proposition 2.

Corollary 1. The $\lambda^{*}$ that satisfies $\Lambda\left(\lambda^{*}, \cdot\right)=0$ has the feature that: $\lambda^{*}$ increases as either the quality of private information, $\pi_{\xi}$, the precision of the supply of the risky asset available for trade, $\pi_{x}$, or the fraction of informed investors in the economy, $\alpha$, increases; $\lambda^{*}$ decreases as the quality of public information, $\pi_{\varepsilon}$, increases; and $\lambda^{*}$ can either increase or decrease as investors' risk tolerance, $\tau$, per-capita risk associated with the common factor, $\sigma_{f}^{2}$, or factor-beta, $\beta$, increases.

Corollary 2. An increase in $\pi_{\varepsilon}$ reduces the asset's risk premium, whereas an increase in $\pi_{\xi}$ increases the risk premium.

Unlike the benchmark case considered in Section 2, Proposition 2 establishes that the effects of $\pi_{\varepsilon}$ and $\pi_{\xi}$ on the risk premium in eqn. (23) are not additively separable from the effect of the common factor. Because $\pi_{\varepsilon}$ and $\pi_{\xi}$ are not additively separable from the common factor and risk associated with the common factor grows with the economy (i.e., 
$\left.\sigma_{F}^{2}=N \sigma_{f}^{2}\right), \pi_{\varepsilon}$ and $\pi_{\xi}$ - despite being finite - will continue to affect the risk premium as $N \rightarrow \infty$. As such, the risk premium is a function of both "traditional" features of the risk premium (i.e., $\tau^{-1} \beta^{2} \sigma_{f}^{2}$ ) and idiosyncratic features (i.e., $\pi_{\varepsilon}$ and $\pi_{\xi}$ ).

In our model the qualities of public and private information operate through $\pi_{\varepsilon}$ and $\pi_{\xi}$, respectively. Increases in the quality of public information increase $\pi_{\varepsilon}$, and increases in the intermediary's private information increase $\pi_{\xi}$. Thus, eqn. (23) establishes that the quality of public and private information affect the risk premium despite the economy having a large number of investors. Eqn. (23) also establishes that $\pi_{\varepsilon}$ and $\pi_{\xi}$ affect the risk premium incremental to the the risky asset's covariance with the common factor, $\beta$. This implies that the qualities of public and private information should manifest in asset prices incremental to factor-beta(s). Finally, because $\pi_{\varepsilon}$ and $\pi_{\xi}$ are asset-specific characteristics, eqn. (23) implies that the risk premium is a function of both factor-beta(s), $\beta$, and asset- (or firm-) specific characteristics.

As has been discussed, when each investor in the economy trades on his own account - as opposed to some fraction delegating their trades - market competition will be perfect. It is a straightforward exercise to show that in the comparable perfect competition setting that results from a fraction $\alpha$ of informed investors trading on their own account, the risk premium will be lower: in effect, the risk premium will reduce to $\tau^{-1} \beta^{2} \sigma_{f}^{2}$, where $\tau^{-1} \beta^{2} \sigma_{f}^{2} \leq$ $\Delta\left(\lambda^{*}, \cdot \cdot{ }^{10}\right.$ In other words, when imperfect competition arises as a consequence of some investors delegating their trades to an intermediary who has private information about $\tilde{\varepsilon}$, the risky asset's risk premium will be higher than in the comparable perfect-competition setting. In Appendix B we prove the following additional corollary to Proposition 2.

Corollary 3. The asset's risk premium will be higher than in a comparable setting where competition among investors is perfect.

\footnotetext{
${ }^{10}$ However, when $\alpha=0$, the risk premium reduces to $\tau^{-1} \beta^{2} \sigma_{f}^{2}$ irrespective of delegation or self-direction: see the discussion "An implication of $\alpha=0$ " subsequent to the proof to Lemma 1 in Appendix C.
} 


\section{Endogenous delegated trade}

\subsection{Discussion}

In this section we extend the model in Section 3 to allow the following elements to be endogenous: (i) the fraction of investors who delegate their trades; (ii) the number of intermediaries; and (iii) the fee that intermediaries charge their clients. Our approach to introducing endogeneity is guided by the analysis in Grossman and Stiglitz (1980) (hereafter GS). Specifically, GS posit an economy of atomistic investors, each of whom can choose to pay a fixed fee and acquire private information. GS show that in equilibrium each investor is indifferent between paying the fee and being informed, versus not paying the fee and remaining uninformed, such that (in equilibrium) there are no rents to acquiring private information. Rather than have investors pay a fee to acquire private information, we assume that investors pay a fee to delegate their trading decisions to a privately informed financial intermediary, where the fee is endogenously determined by competition among intermediaries.

The central premise of GS is that the cost of acquiring private information about a firm (or risky asset more generally) is sufficiently small such that it is financially viable for an individual investor to bear the full cost of acquiring private information. Alternatively, one could imagine that the cost is so large that no single, individual investor could possibly bear the full cost of acquiring private information. Delegated trade allows the individual investor to bear a fraction of the information acquisition cost, by paying a small fee to a privately informed financial intermediary. In return, the intermediary does not share his private information with his clients, but rather trades on their behalf based on the information. In this regard, the role of the intermediary in the economy is to acquire private information, trade on behalf of his clients, and charge each client a very small fee. The collection of small fees reimburses the intermediary for large information acquisition costs. This model of 
information acquisition, where costs are spread among a collection of very small investors, offers an alternative story about how information is gathered and disseminated in a large economy. ${ }^{11}$

While our approach to allowing delegated trade to be endogenous is guided by GS, we face three challenges in applying their analysis. First, in addition to considering rents from investors' decision to delegate versus self-direct, we also need to consider the potential rents of the intermediaries to whom trade is delegated. For tractability, we assume intermediaries are homogenous and each intermediary maximizes profits. Second, whereas GS assume that markets are perfectly competitive, delegated trade results in imperfect competition and modeling imperfect competition is inherently more challenging. Third, the objective of our paper is to distinguish the effect of different types of risks on the risk premium - and this more than anything else complicates our analysis.

As in GS, we focus on an economy with no rents. In an economy with no rents, there are three conditions that hold (in equilibrium) that allow us to solve for the fraction of investors who delegate their trades; the number of intermediaries; and the fee that intermediaries charge their clients. (1) Intermediary profit maximization. We assume that each intermediary earns fee revenue from his clients (per client fee $\times$ number of clients); pays a fix setup cost (e.g., cost of acquiring private information); and bears a variable cost for each additional client (e.g., an administrative cost). (2) Intermediary zero profit condition. In equilibrium, the number of intermediaries is such that each intermediary earns zero profits. If there were profits (losses), additional intermediaries would enter (leave) the market, thereby invalidating the equilibrium. In a frictionless market for delegation, intermediaries earn no rents. (3) The investor indifference condition. In equilibrium, there are no rents to investors from

\footnotetext{
${ }^{11}$ In addition to having access to private information through an intermediary, one could imagine alternative ways investors might become informed including gathering information on their own account. Irrespective of the distribution of private information across investors, our results will be sustained provided that some (positive) fraction of investors delegate their trades to an intermediary: this is the central message of Section 3.
} 
delegation. Conditional on the delegation fee, each investor is indifferent between delegation and self-direction. If this were not the case, there would be gains (losses) to delegation - in which case all investors would (not) delegate.

Finally, it is important to note that allowing various features of the equilibrium to be endogenous does not alter the result that characteristics of the firm's information environment (i.e., $\pi_{\varepsilon}$ and $\pi_{\xi}$ ) continue to manifest in the risk premium incremental to factor-beta(s). Indeed, the expression for the risk premium given by eqn. (23) only changes insofar as the equilibrium fraction of investors who delegate their trades, $\alpha$, is now endogenous and thus depends on the characteristics of the economy.

\subsection{Analysis}

As a preliminary step toward characterizing an economy with no rents, let $\frac{\phi}{N}$ represent the fee each intermediary charges to each of his clients, $M$ the number of intermediaries, $\alpha_{0}$ an individual intermediary's client base expressed as a fraction of the investors in the economy (recall we assume intermediaries are identical), and $\alpha\left(\frac{\phi}{N}\right)$ the total fraction of investors in the economy who choose to delegate their trades to an intermediary as a function of the fee, such that investors are indifferent between delegating and self-directing their trades. Note that $\alpha\left(\frac{\phi}{N}\right) N$ represents the total number of investors in the economy who choose to delegate, whereas $\alpha_{0} N$ denotes the number of clients of each intermediary. By virtue of assuming all intermediaries are identical, $\alpha\left(\frac{\phi}{N}\right)=\alpha_{0} M$. We offer the following result.

Lemma 1. In our characterization of an economy with delegated trade, for any fee of $\frac{\phi}{N}$, where $\phi \in\left[0, \frac{\tau}{2} \frac{\pi_{\xi}}{\pi_{x}} \frac{\pi_{x}+\pi_{\xi}}{\left(\pi_{\varepsilon}+\pi_{\xi}\right)^{2}} \frac{1}{\beta^{2} \sigma_{f}^{2}}\right]$, there exists a fraction $\alpha\left(\frac{\phi}{N}\right)$ of investors in the economy who delegate their trades that leaves all investors indifferent between delegating versus selfdirecting their trades.

While Lemma 1 is ostensibly a straightforward result - perhaps even "obvious" insofar 
as its similarity to the comparable result in GS - it is very challenging to prove: as such we devote an entire appendix, Appendix C, to its proof. The additional complexity of proving Lemma 1 arises from the fact that we posit an imperfect competition setting in conjunction with distinguishing between two types of risks: risks that grow with the economy and risks that do not. Lemma 1 establishes that if investors are charged a fee to delegate their trades, then there exists a fraction of investors in the economy who delegate their trades that leaves all investors indifferent between delegating versus self-directing their trades. Effectively, no investor - either those who delegate or those who self-direct - earns any rents when the fee for delegation is $\frac{\phi}{N}$.

Now we turn our attention to the intermediaries. We assume an intermediary does not trade, but instead gathers information about the risky asset and then coordinates the trades of his clients. We assume that information gathering entails a fixed cost $K$, and coordinating trade for a fraction $\alpha_{0}$ of investors in the economy entails a variable cost $\frac{1}{2} k\left(\alpha_{0}\right)^{2}$. The latter ensures that an intermediary's optimization problem as a function of $\alpha_{0}$ is concave (i.e., marginal profits decline as $\alpha_{0}$ increases), and thus its solution is unique and well defined.

Each intermediary conjectures that other intermediaries will charge each client a fee of $\frac{\hat{\phi}}{N}$, where the caret (i.e., $\hat{\phi}$ ) implies a conjecture (as opposed to an equilibrium outcome). Hence, the intermediary charges $\frac{\hat{\phi}}{N}$ : charging more than $\frac{\hat{\phi}}{N}$ will result in no clients, and charging less than $\frac{\hat{\phi}}{N}$ will result in having as clients every investor who wants to delegate (which is some fraction of investors greater than $\alpha\left(\frac{\hat{\phi}}{N}\right)$ ), and these many clients are too many because the (variable) cost of coordinating clients is increasing at an increasing rate. ${ }^{12}$ While each client's fee, $\frac{\hat{\phi}}{N}$, becomes asymptotically very small as the economy becomes large (i.e., $N \rightarrow \infty$ ), each intermediary's number of clients, $\alpha_{0} N$, becomes correspondingly large; these have offsetting effects on an intermediary's revenue such that $\alpha_{0} N \cdot \frac{\hat{\phi}}{N}=\alpha_{0} \cdot \hat{\phi}$ describes

\footnotetext{
12 This will always be the case in the equilibrium we posit in Proposition 3, where at least one person or institution serves as an intermediary.
} 
his revenue. Thus, an intermediary's profit is given by:

$$
\alpha_{0} \cdot \hat{\phi}-\frac{1}{2} k\left(\alpha_{0}\right)^{2}-K
$$

Maximizing eqn. (24) with respect to $\alpha_{0}$ yields $\alpha_{0}=\frac{\hat{\phi}}{k}$, or $\hat{\phi}=k \alpha_{0}$. Assuming the zero-profit condition and substituting this expression into eqn. (24) implies that an intermediary's profit is given by

$$
\frac{1}{2} k \alpha_{0}^{2}-K=0
$$

Recall from Lemma 1 that if the fee is $\frac{\hat{\phi}}{N}$ then the total fraction of investors who will choose to delegate their trades is $\alpha\left(\frac{\hat{\phi}}{N}\right)$, and because all intermediaries are identical $\alpha_{0}=\frac{\alpha\left(\frac{\hat{\phi}}{N}\right)}{M}$. Thus, the equilibrium number of intermediaries must satisfy

$$
\frac{1}{2} k\left(\frac{\alpha\left(\frac{\hat{\phi}}{N}\right)}{M}\right)^{2}-K=0
$$

or $M=\sqrt{\frac{k}{2 K}} \alpha\left(\frac{\hat{\phi}}{N}\right)$.

As an aside, for there to be at least one person or institution willing to serve as an intermediary, it must be the case that $M \geq 1$, which, in turn, requires $\alpha\left(\frac{\hat{\phi}}{N}\right) \geq \sqrt{2 \frac{K}{k}}$. Additionally, $\alpha\left(\frac{\hat{\phi}}{N}\right)$ cannot exceed 1. Thus, in conjunction with assuming $\alpha\left(\frac{\hat{\phi}}{N}\right) \geq \sqrt{2 \frac{K}{k}}$ we are also required to assume $k \geq 2 K$.

Finally, recall that $\hat{\phi}=k \alpha_{0}$, and thus for the conjectured fee to be an equilibrium outcome it must be the case that

$$
\hat{\phi}=k \alpha_{0}=k \frac{\alpha\left(\frac{\hat{\phi}}{N}\right)}{M}=\sqrt{2 k K}
$$

This implies the following result, the proof of which should be clear from Lemma 1 and the discussion above. 
Proposition 3. The following elements constitute an equilibrium in an economy where no intermediary or investor earns any rents and the fraction of investors who delegate their trades, the number of intermediaries, and the fee that intermediaries charge their clients are all endogenous. The total fraction of investors in the economy who delegate their trades to an intermediary is $\alpha\left(\frac{\sqrt{2 k K}}{N}\right)$; there are $M=\sqrt{\frac{k}{2 K}} \alpha\left(\frac{\sqrt{2 k K}}{N}\right)$ intermediaries in the economy; intermediaries charge each client a fee of $\frac{\sqrt{2 k K}}{N}$; where we require $\alpha\left(\frac{\sqrt{2 k K}}{N}\right) \geq \sqrt{2 \frac{K}{k}}$ (and thus, in effect, $k \geq 2 K$ ) to ensure the existence of at least one intermediary, and

$$
\sqrt{2 k K}<\frac{\tau}{2} \frac{\pi_{\xi}}{\pi_{x}} \frac{\pi_{x}+\pi_{\xi}}{\left(\pi_{\varepsilon}+\pi_{\xi}\right)^{2}} \frac{1}{\beta^{2} \sigma_{f}^{2}}
$$

to ensure that their exists a fraction $\alpha\left(\frac{\sqrt{2 k K}}{N}\right)$ of investors in the economy who are indifferent between delegating and self-directing their trades.

\subsection{Numerical comparative statics}

In this section we illustrate graphically the effects of changes in the quality of public information $\left(\pi_{\varepsilon}\right)$, the quality of private information $\left(\pi_{\xi}\right)$, systematic cash flow volatility $\left(\sigma_{f}\right)$, and the numerator of the delegation fee $(\phi)$ on: the fraction of investors who choose to delegate their trades $(\alpha)$; the level of market illiquidity $(\lambda)$; and the risky asset's risk premium $(\Delta) .{ }^{13}$ To facilitate the analysis, henceforth (and without loss of generality) we fix the remaining exogenous parameters at 1 : that is, we assume $\beta=\tau=\pi_{x}=1 .^{14}$ In our illustrations, $\alpha$ solves for $\Omega\left(\lambda, \alpha, \beta, \tau, \sigma_{f}, \pi_{\varepsilon}, \pi_{\xi}, \pi_{x}\right)+2 \frac{\phi}{\tau}=0$, where $\Omega(\lambda, \cdot)$ is as defined in eqn. (36) in Appendix $\mathrm{C} ; \lambda$ solves for $\Lambda(\lambda, \cdot)=0$, where $\Lambda(\lambda, \cdot)$ is as defined in the Proof to Proposition

\footnotetext{
13 From Proposition $3, \phi$ must satisfy the requirement that $\phi=\sqrt{2 k K}$; nonethless, $k$ and $K$ can be selected arbitrarily such that, for all intents and purposes, $\phi$ can be treated as an exogenous parameter, subject to the requirement in Lemma 1 that $\phi$ falls within an appropriate range to guarantee the existence of an equilibrium $\alpha$.

${ }^{14}$ In our model the distinciton between $\beta$ and $\sigma_{f}$ is arbitrary, and so identical results hold if $\sigma_{f}=1$ and $\beta$ is allowed to vary.
} 
2 in Appendix B; and $\Delta(\lambda, \cdot)$ is as defined in eqn. (23) in Section 3.

We generate three sets of graphs, Figures 1, 2, and 3, where each figure contains three panels: Panel A graphs $\alpha$, Panel B graphs $\lambda$, and Panel $\mathrm{C}$ graphs $\Delta$. Figure 1 examines the interaction of public and private information on $\alpha, \lambda$, and $\Delta$, when $\pi_{\varepsilon} \in(0,1]$ and $\pi_{\xi} \in(0,1]$ (setting $\sigma_{f}=1$ and $\phi=0.03$ ). [INSERT FIGURE 1 HERE.] Figure 2 examines the interaction of public information and systematic cash flow volatility on $\alpha, \lambda$, and $\Delta$, when $\pi_{\varepsilon} \in(0,1]$ and $\sigma_{f} \in(0,1]$ (setting $\pi_{\xi}=1$ and $\phi=0.03$ ). [INSERT FIGURE 2 HERE.] Figure 3 examines the interaction of public information and the delegation fee on $\alpha, \lambda$, and $\Delta$, when $\pi_{\varepsilon} \in(0,1]$ and $\phi \in\left(0,0.20\right.$ ] (setting $\sigma_{f}=1$ and $\left.\pi_{\xi}=1\right)$. [INSERT FIGURE 3 HERE.] Collectively, Figures 1-3 document that the fraction of investors who choose to delegate their trades $(\alpha)$, the level of illiquidity $(\lambda)$, and the risky asset's risk premium $(\Delta)$, all decrease as the quality of public information $\left(\pi_{\varepsilon}\right)$ increases.

Another interesting relation is the one between the cost of delegation and price informativeness: the efficiency with which price communicates intermediaries' private information to uninformed investors. As in GS, price in our analysis does not perfectly communicate intermediaries' private information; otherwise, there would be no incentive to delegate trade. One straightforward metric to measure price informativeness is the precision of the information uninformed investors glean by conditioning their beliefs on price, $\pi_{\delta}$ : see eqn. (11). An increase in the delegation fee has two countervailing effects on $\pi_{\delta}$. First, an increase in the fee results in a decrease in the fraction of investors who delegate their trades (i.e., decreases $\alpha$ ); this works to reduce the informativeness of price because a smaller fraction of investors in the economy is informed. Second, an increase in the fee results in a decrease in illiquidity (i.e., decreases $\lambda$ ) because fewer investors are informed; this works to increase the informativeness of price. Consequently, whether an increase in the delegation fee is associated with an increase or a decrease in the informativeness of price depends on whether the $\alpha$-effect versus the $\lambda$-effect dominates. In Figure 4 we graphically illustrate the effect of the 
delegation fee $(\phi)$ on price informativeness $\left(\pi_{\delta}\right)$ for the same numerical values used earlier (i.e., $\phi \in(0,0.20]$ and all remaining exogenous parameters set to 1$)$. [INSERT FIGURE 4 HERE.] Figure 4 shows that price informativeness decreases as the delegation fee increases.

\section{Capital market where the common factor is tradable}

It is common practice in the noisy rational expectations literature to consider an economy with a single risky asset and a risk-free bond. (e.g., Grossman and Stiglitz, 1980; Kyle, 1989; Admati and Pfleiderer, 1990). This is not to suggest the insights from such models do not apply to economies populated by multiple risky assets (e.g., Admati, 1985). Rather, the complexity associated with including multiple risky assets in a model of strategic trade is so great, and the additional insight so small, that it is common practice to model price formation and strategic trade for a single risky asset. In our context, the focus on a single risky asset considerably facilitates the discussion.

Nonetheless, to provide insight on this issue without burdening the model with unnecessary complexity, in this section we consider the case where (as in Section 3) an exogenous fraction $\alpha$ of investors delegate their trades in a risky asset, but can also trade a noisy measure of the common factor. For example, in our model $\tilde{F}$ represents the true common factor; as such, one can think of $\tilde{F}$ as a macroeconomic state variable that determines future investment opportunities. The true common factor, however, is not necessarily a traded security (Merton, 1973). We capture this phenomenon by allowing for the possibility that the true common factor can only be traded with noise. Here we show that our results depend on whether investors can decompose and price separately the risk associated with the systematic and the idiosyncratic components of the risky asset's cash flow, and not the number of tradeable securities in the economy.

In principle, diversifying into the common factor allows investors to decompose the risks 
associated with the systematic and the idiosyncratic components of the risky asset's cash flow. For example, if the traded common factor tracks the true common factor without noise, investors can separate out the idiosyncratic component of the risky asset's cash flow, $\tilde{\varepsilon}$, by going long shares of the risky asset and short $\beta$ times the common factor. The variance of the payoffs to this portfolio will simply be $\sigma_{\varepsilon}^{2}$, the variance of the idiosyncratic cash flow. Because investors can isolate these two components, only risk associated with the systematic component will be priced. ${ }^{15}$ While this may seem the end of the story, it comes with an important caveat. If investors can only trade a noisy measure of the true common factor, investors will be unable to perfectly decompose cash flow (i.e., the variance of payoffs to the aforementioned portfolio is not $\left.\sigma_{\varepsilon}^{2}\right)$. In this circumstance, our results hold.

We illustrate this by first showing that if the traded common factor tracks the true common factor without noise, investors can use the traded factor to perfectly decompose the systematic and idiosyncratic components, such that only risk associated with the systematic component of cash flow affects the risk premium. Alternatively, we show that if investors can only trade a noisy measure of the true common factor, investors will be unable to perfectly decompose the risky asset's systematic and idiosyncratic components, such that the risk premium will depend on $\pi_{\varepsilon}$ and $\pi_{\xi}$. Because the analysis in this section is qualitatively identical to the analysis in Section 3, we confine most of it to Appendix D.

Recall that we represent the risky asset's cash flow by $\tilde{V}_{a}=\mu_{a}+\beta \tilde{F}+\tilde{\varepsilon}$, where $\tilde{F}$ has a normal distribution with mean 0 and variance $\sigma_{F}^{2}=N \sigma_{f}^{2}$ and $\tilde{\varepsilon}$ has a normal distribution with mean 0 and precision $\pi_{\varepsilon}$. We represent the cash flow to the traded common factor, $\tilde{V}_{T}$, by $\tilde{V}_{T}=\tilde{F}+\tilde{\mu}_{T}$, where $\tilde{\mu}_{T}$ represents investors' uncertain assessment of the expected cash flow: $\tilde{\mu}_{T}$ is an independent random variable with a normal distribution with mean $\mu$ and

\footnotetext{
15 This result is not surprising. If cash flows are assumed to follow a factor structure and the true common factor is traded without noise, then by assumption (in a large economy with rational investors) only the true common factor and investors' risk-aversion will determine asset prices. Indjejikian (2007) makes a similar point in the context of Lambert et al. (2007).
} 
variance $\sigma_{T}^{2}$, where $\sigma_{T}^{2}=N \sigma_{\mu}^{2}$. As there is no private information about the common factor, we assume that all investors act as price takers in their demands for the traded common factor. As in Section 3, uninformed investors act as price takers in their demands for the risky asset, whereas an intermediary trades in behalf of informed investors. In Appendix D we prove the following proposition.

Proposition 4. In the absence of noise in the traded common factor, the risky asset's risk premium is independent of $\pi_{\varepsilon}$ and $\pi_{\xi}$. Alternatively, in the presence of noise in the traded factor, the risky asset's risk premium is positive and a function of $\pi_{\varepsilon}$ and $\pi_{\xi}$.

Proposition 4 provides our main result for a setting where investors can trade both the risky asset and the common factor. In the absence of noise in the traded factor, investors can use the common factor to perfectly decompose the risky asset's systematic and idiosyncratic components such that as the economy becomes large only the former is priced. In the presence of noise in the traded factor, however, this is not the case. Here, the risk premium will be positive and a function of $\sigma_{f}^{2}, \sigma_{\mu}^{2}, \pi_{\varepsilon}$ and $\pi_{\xi}$. This result points to the fact that considerations of whether our analysis extends more generally should be based on the ability of the market to decompose and separately price risks - not on the number of assets that are incorporated into the economy. ${ }^{16}$

Given that our results are predicated on the notion that investors can only trade a noisy measure of the true common factor, it is instructive to consider how our results comport with the perception that the observable market portfolio is the true common factor; a perception premised on intuition from the CAPM. In this regard, we make two points. First, Roll (1977) points out that the theoretical market portfolio implied by the CAPM is - in practice - not observable or tradable: it includes an infinite number of securities, including those related to

\footnotetext{
16 The benchmark model discussed in Section 2, which is otherwise identical to our main model in Section 3, makes clear that our results do not attain when all trade is self-directed. This serves to emphasize that our results are driven by delegation, and not some other feature of the economy, such as the number of assets available for trade.
} 
private companies. Second, because all investors in the CAPM are homogenous, it is optimal for all investors to hold the same portfolio (the market portfolio), hence all securities are priced relative to that portfolio. In contrast, in models of heterogeneous information, each investor's optimal portfolio depends on his information set. Consequently, not all investors hold the market portfolio, and thus it is not feasible to price securities exclusively in terms of the market portfolio (e.g., Biais et al., 2010). For these reasons, we caution against trying to extend economic intuition based on a model of homogenous beliefs (the CAPM) to a model of heterogeneous beliefs, and caution against interpreting the common factors frequently used in empirical asset pricing tests (e.g., the CRSP market portfolio and the Fama-French factors) as noiseless measures of the true common factors.

\section{Empirical Implications}

Our primary results suggest that delegated trade inhibits a large economy from becoming perfectly competitive, and, as a consequence, characteristics of the firm's information environment manifest in asset prices incremental to the firm's factor-beta despite the economy being large. For example, unlike the traditional expression for the risk premium in a large economy (which depends exclusively on investors' risk tolerance, the firm's factor-beta, and systematic cash flow volatility: see Section 2.1), the expression for the risk premium in our model also includes firm characteristics related to the information environment (notably $\pi_{\varepsilon}$ and $\pi_{\xi}$ : see Section 3.5). This result has several implications for empirical work examining the relation between the information environment and expected returns.

First, to the extent that a particular firm characteristic affects the quality of public information, the quality of private information, or the firm's idiosyncratic cash flow volatility, our analysis suggests this characteristic will be priced incremental to the firm's factor-beta. In this regard, our results suggest a host of firm characteristics that could potentially affect 
expected returns incremental to factor-betas. Thus, while we interpret some of the predictions of the model primarily from the perspective of accounting information, the predictions can be thought of as applying more broadly to any source of public or private information and/or idiosyncratic cash flow volatility.

Second, because risks manifest in the form of both firm characteristics and factor-betas, our analysis suggests factor-based asset pricing tests cannot rule out that a particular risk is priced - only that it is priced in the form of a common risk factor. This is particularly relevant for the ongoing (empirical) debate about the pricing of accounting information. ${ }^{17}$ While much of the empirical literature focuses on testing whether the quality of accounting information represents a separate common risk factor, our results suggest that the focus on a separate, accounting quality risk factor is too limiting. In particular, our results suggest that the quality of a firm's accounting information can affect expected returns as an individual firm-level characteristic (e.g., through $\pi_{\varepsilon}$ ) even if accounting quality is not priced as a separate common factor. Thus, our model suggests that a firm's accounting quality can be priced as an additional risk, even if the factor-beta on an accounting quality risk factor is not priced (e.g., Core et al., 2008).

One avenue for future empirical research on this topic might be to explore whether accounting quality is priced as a firm characteristic (as our results would suggest) or as a separate common risk factor (as suggested by Kim and Qi, 2010; Ogneva, 2012). Prior work in finance has explored a similar question as it relates to whether size, book-to-market, and accruals are priced as systematic risk factors or as firm characteristics (e.g., Daniel and Titman, 1997; Davis et al., 2000; Hirshleifer et al., 2012). ${ }^{18}$ If accounting quality is priced

\footnotetext{
17 See Francis et al. (2005), Core et al. (2008), Kim and Qi (2010), and Ogneva (2012). For a review see Shevlin (2013).

18 Studies in this literature run an empirical horse-race between the predictive abilities of factor-betas versus firm characteristics for a cross-section of returns. For example, within the context of the debate over whether size and book-to-market are priced as factors or as characteristics, prior work regresses expected returns on estimates of the firm's factor-betas with respect to the Fama-French market, size, and book-to-
} 
empirically as a separate common risk factor, then not only should the accounting quality factor-beta be related to expected returns, but it should be related to expected returns incremental to the accounting quality characteristic. In contrast, if accounting quality is priced empirically as a firm characteristic, then the firm-level accounting quality characteristic should be related to expected returns, and should subsume the explanatory power of accounting quality factor-beta.

Third, much of the existing literature examines the "on average" relation between the quality of public information, illiquidity, and expected returns either across countries or across firms (within a given country). While our results suggest this relation is positive, the results also suggest considerable cross-sectional variation in the effect of public information. For example, our numerical analysis illustrates various "cross-partials" regarding the effect of public information $\left(\pi_{\varepsilon}\right)$ on illiquidity $(\lambda)$ and the risk premium $(\Delta)$. Figure 1 illustrates how the effect of public information varies with private information, Figure 2 illustrates how the effect of public information varies with the level of systematic cash flow volatility, and Figure 3 illustrates how the effect of public information varies with the costs borne by the intermediary through the delegation fee he charges. Perhaps, most notably, Figure 2 shows that the effect of public information on the risk premium vanishes as systematic cash flow volatility approaches zero. Thus, while some studies have examined whether the pricing of public information varies with market competition (e.g., Armstrong et al., 2011; Akins et al., 2012; Bhattacharya et al., 2012), our results suggests additional variation in the pricing of public information. Empirical researchers can potentially use these theoretical results to develop more powerful tests of the empirical relation among public information, illiquidity, and expected returns. ${ }^{19}$

market factors $\left(\hat{\beta}^{M K T R F}, \hat{\beta}^{S M B}\right.$, and $\hat{\beta}^{H M L}$, respectively), and additionally includes measures of the firm's
size and book-to-market ratio in the regression. Studies in this literature generally find that the coefficients
on firm size and book-to-market are significantly different from zero, and the coefficients on the estimated
Fama-French factor-betas are insignificantly different from zero.
${ }^{19}$ For example, Lang et al. (2012) examine the relation between firm-level measures of transparency and 
Finally, we hasten to add three caveats that future empirical tests will need to confront in order to draw valid inferences.

(1) Construct validity. Our predictions are premised on the notion that the empirical researcher has a valid empirical measure of the theoretical construct of interest. In this regard, our predictions are stated explicitly in terms of constructs of interest rather than common empirical measures of these constructs. For example, we state our predictions in terms of "accounting quality" as a construct. Our model is one of information economics and not measurement - the accounting process itself is outside the scope of our model. Consequently, the model does not speak to how one determines the "best" empirical measure of an accounting-quality construct. Empirical measurement choices require researchers to make tradeoffs not considered in this paper (e.g., bias, measurement error, etc.). In this regard, any empirical tests of our model are necessarily joint tests of our theoretical predictions and the presumption that a specific empirical proxy measures the theoretical construct of interest (Zimmerman, 2013).

(2) Disentangling the effect of real volatility of cash flows from the effect of public information. Recall from Section 2 that $\pi_{\varepsilon}$ represents investors' assessment of idiosyncratic cash flow volatility, which is driven by the sum of two (idiosyncratic) forces: the real volatility of cash flow and common knowledge about that cash flow. This implies that either a reduction in real volatility or an increase in the quality of public information about volatility works to increase $\pi_{\varepsilon}$. To the extent that an empirical researcher wants to make specific statements about the quality of public information, separate and apart from the real volatility of cash flow, it is important that the researcher employs a design that cleanly distinguishes between

illiquidity across 46 countries, distinguishing between periods when the volatility of a country's stock market is high versus low. Lang et al. (2012) find the effect of transparency on illiquidity is most pronounced in periods when the volatility of the country's stock market is high. Viewing firm transparency as a measure of the quality of public information $\left(\pi_{\varepsilon}\right)$ and volatility of a country's stock market as systematic volatility $\left(\sigma_{f}\right)$, the results are consistent with the notion that the effect of public information on illiquidity $(\lambda)$ is largest when systematic volatility is high (e.g., Figure 2, Panel B). 
the two. For example, consider a circumstance where a researcher employs an empirical measure of accounting quality that commingles volatility of cash flow and the quality of public information. This is not an issue for testing the predictions of our model, per se, because in our model both of these constructs affect illiquidity $(\lambda)$ and the risk premium $(\Delta)$ identically: irrespective of whether the measure reflects the volatility of cash flow or quality of public information, the predictions would be the same. However, the inferences that one can draw from such an empirical design are limited. If the empirical design commingles volatility of cash flow and quality of public information, inferences cannot be unambiguously attributed to the effect of information (as distinct form the effect of cash flow volatility).

(3) Endogeneity. While we predict that delegated trade makes markets imperfect, giving rise to the relations we document, delegated trade, illiquidity, and expected returns are endogenous. This confounds the interpretation of empirical tests that relate measures of delegated trade to illiquidity and expected returns. For example, the equilibrium level of delegated trade is itself a function of the information environment (which is exogenous). Panel A in each of Figures 1 through 3 graphically illustrates how the level of delegated trade varies with the qualities of public and private information, systematic cash flow volatility, and the information acquisition costs borne by the intermediary through the delegation fee he charges. Thus, in addition to informing future empirical work on the relation between the information environment and expected returns, our results can also potentially inform future empirical work on the relation between the information environment and the extent to which individual investors delegate their trades.

\section{Conclusion}

We extend a standard, rational expectations model of trade to incorporate the possibility that individual investors can delegate their trades to a privately informed financial intermediary: 
for example, an institutional investor. We motivate this modeling approach by appealing to the notion that the cost of acquiring private information (not already impounded in prices) is so large that it is not feasible for an individual investor to bear the entirety of this cost. Instead, the role of an intermediary is to acquire private information, trade on behalf of his clients, and charge each individual client a very small fee as compensation for his services. The collection of small fees reimburses the intermediary for large information acquisition costs, and allows the costs of information acquisition to be spread across multiple investors.

It is easy to imagine a circumstance where the risk premium is characterized exclusively in terms of factor-betas (e.g., the CAPM). However, given the mounting empirical evidence that firm characteristics affect asset prices, the purpose of this paper is to identify and examine a circumstance in which characteristics of the firm's information environment are priced incremental to factor-betas despite the economy being large. Specifically, we show that the risk premium is a function of a firm's exposure to a common risk factor and three idiosyncratic firm characteristics: (i) the quality of public information; (ii) the quality of private information; and (iii) the firm's idiosyncratic cash flow volatility.

Large economies typically imply that markets are perfectly competitive and, for this reason, characteristics of the firm's information environment are not thought to affect a firm's risk premium incremental to common risk factors. However, the presence of an intermediary implies that imperfect competition is sustained despite the fact that the economy is large. As the number of investors in the economy becomes large, an intermediary's client base grows in tandem. Thus, even though the impact of any single investor's demand on price declines as the economy grows, an intermediary's effect on price is sustained. The ongoing presence of this market imperfection prevents the large economy from becoming perfectly competitive, and ensures that characteristics of the firm's information environment continue to manifest in the risk premium. 


\section{References}

Admati, A., 1985. A noisy rational expectations equilibrium for multi-asset securities markets. Econometrica 53, 629-658.

Admati, A., Pfleiderer, P., 1990. Direct and indirect sales of information. Econometrica 58, 901-928.

Akins, B., Ng, J., Verdi, R., 2012. Investor competition over information and the pricing of information asymmetry. The Accounting Review 81, 35-58.

Armstrong, C., Core, J., Taylor, D., Verrecchia, R., 2011. When does information asymmetry affect the cost of capital? Journal of Accounting Research 49, 1-40.

Bertomeu, J., Cheynel, E., 2014. Disclosure and the cost of capital: a survey of the theoretical literature. Working paper.

Bhattcharya, N., Ecker, F., Olsson, P., Schipper, K., 2012. Direct and mediated associations among earnings quality, information asymmetry, and the cost of equity. The Accounting Review 87, 449-482.

Biais, B., Bossaerts, P., Spatt, C., 2010. Equilibrium asset pricing and portfolio choice under asymmetric information. Review of Financial Studies 23, 1503-1543.

Caskey, J., Hughes, J., Liu, J., 2012. Strategic informed trades, diversification, and expected returns. Working paper.

Chen, Q., Huang, Z., Zhang, Y., 2014. The effects of public information with asymmetrically informed short-horizon investors. Journal of Accounting Research 52, 635-669.

Clinch, G., 2013. Disclosure quality, diversification and the cost of capital. Australian Journal of Management 38, 475-489.

Core, J., Guay, W., Verdi, R., 2008. Is accruals quality a priced risk factor? Journal of Accounting and Economics 46, 2-22.

Cuoco, D., Kaniel, R., 2011. Equilibrium prices in the presence of delegated portfolio management. Journal of Financial Economics 101, 264-296.

Daniel, K., Hirshleifer, D., Subrahmanyam, A., 2001. Overconfidence, arbitrage, and equilibrium asset pricing. Journal of Finance 56, 921-965.

Daniel, K., Hirshleifer, D., Subrahmanyam, A., 2005. Investor psychology and tests of factor pricing models. Working paper. 
Daniel, K., Titman, S., 1997. Evidence on the characteristics of cross sectional variation in stock returns. Journal of Finance 52, 1-33.

Davis, J., Fama, E., French, K., 2000. Characteristics, covariances, and average returns: 1929 to 1997. Journal of Finance 55, 389-406.

Fama, E., 1976. Foundations of Finance. New York: Basic Books.

Francis, J., LaFond, R., Olsson, P., Schipper, K., 2005. The market pricing of accruals quality. Journal of Accounting and Economics 39, 295-327.

French, K., 2008. Presidential address: The cost of active investing. Journal of Finance 63, 1537-1573.

Gao, P., 2010. Disclosure quality, cost of capital, and investor welfare. The Accounting Review 85, 1-29.

Garcia, D., Vanden, J., 2009. Information acquisition and mutual funds. Journal of Economic Theory 144, 1965-1995.

Gomes, J., Kogan, L., Zhang, L., 2003. Equilibrium cross section of returns. Journal of Political Economy 111, 693-732.

Grossman, S., Stiglitz, J., 1980. On the impossibility of informationally efficient markets. American Economic Review 70, 393-408.

Hirshleifer, D., Hou, K., Teoh, S., 2012. The accrual anomaly: Risk or mispricing? Management Science 58, 320-335.

Hughes, J., Liu, J., Liu, J., 2007. Information asymmetry, diversification, and the cost of capital. The Accounting Review 82, 705-729.

Indjejikian, I., 2007. Discussion of accounting information, disclosure, and the cost of capital. Journal of Accounting Research 45, 421-426.

Kim, D., Qi., Y., 2010. Accruals quality, stock returns, and macroeconomic conditions. The Accounting Review 85, 937-978.

Kyle, A., 1989. Informed speculation with imperfect competition. Review of Economic Studies 56, 317-355.

Kyle, A., Ou-Yang, H., Wei, B., 2011. A model of portfolio delegation and strategic trading. Review of Financial Studies 24, 3778-3812.

Lambert, R., Leuz, C., Verrecchia, R., 2007. Accounting information, disclosure, and the cost of capital. Journal of Accounting Research 45(2), 385-420. 
Lambert, R., Leuz, C., Verrecchia, R., 2012. Information asymmetry, information precision, and the cost of capital. Review of Finance 16, 1-29.

Lambert, R., Verrecchia, R., 2014. Information, Illiquidity, and Cost of Capital. Contemporary Accounting Review (forthcoming).

Lang, M., Lins, K., Maffett, M., 2012. Transparency, liquidity, and valuation: International evidence on when transparency matters most. Journal of Accounting Research 50, 729-774.

Merton, R., 1973. An intertemporal capital asset pricing model. Econometrica 41, 867-887.

Ogneva, M., 2012. Accrual quality, realized returns, and expected returns: The importance of controlling for cash flow shocks. The Accounting Review 87, 1415-1444.

Roll, R., 1977. A critique of the asset pricing theory's tests. Journal of Financial Economics 4, 129-176.

Shevlin, T., 2013. Some personal observations on the debate on the link between financial reporting quality and the cost of equity capital. Australian Journal of Management 38, 447-473.

Zimmerman, J., 2013. Myth: external financial reporting quality has a first-order effect on firm value, Accounting Horizons 27, 887-894. 
Figure 1. Effect of Public and Private Information

Panel A. Delegated Trade

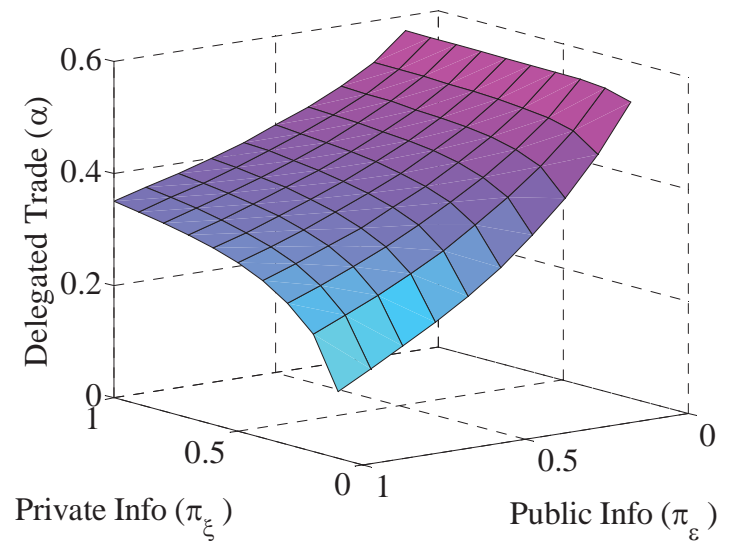

Panel B. Illiquidity

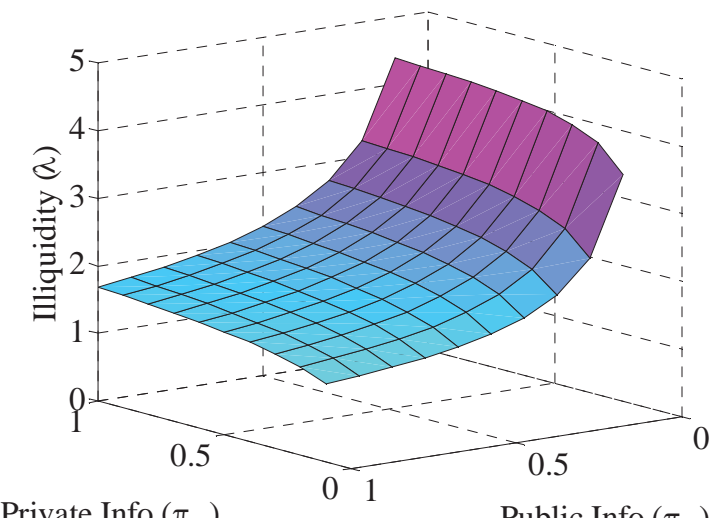

Private Info $\left(\pi_{\xi}\right)$

Public Info $\left(\pi_{\varepsilon}\right)$

\section{Panel C. Risk Premium}

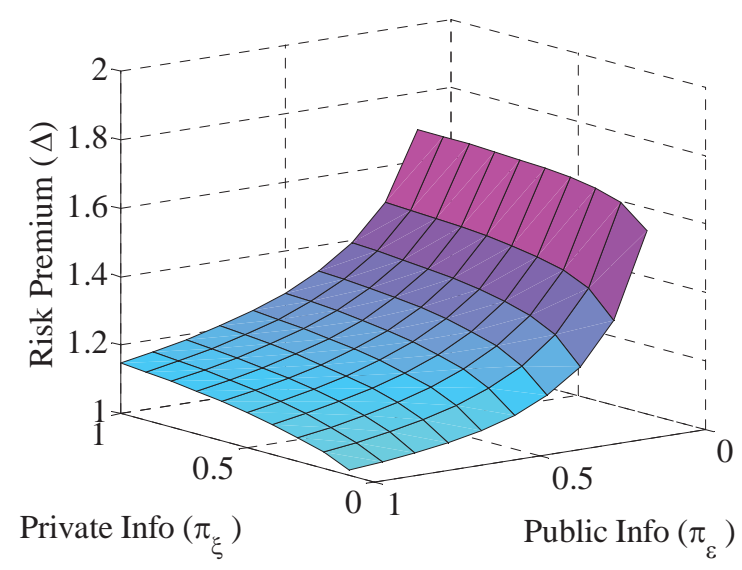

Panel A plots the relation among delegated trade $(\alpha)$, public information $\left(\pi_{\varepsilon}\right)$, and private information $\left(\pi_{\xi}\right)$. Panel B plots the relation among illiquidity $(\lambda)$, public information $\left(\pi_{\varepsilon}\right)$, and private information $\left(\pi_{\xi}\right)$. Panel C plots the relation among the risk premium $(\Delta)$, public information $\left(\pi_{\varepsilon}\right)$, and private information $\left(\pi_{\xi}\right)$. In each panel, the $y$-axis is increasing bottom-to-top, and the $x$ - and z-axes are increasing right-to-left. 
Figure 2. Effect of Public Information and Systematic Cash Flow Volatility

Panel A. Delegated Trade

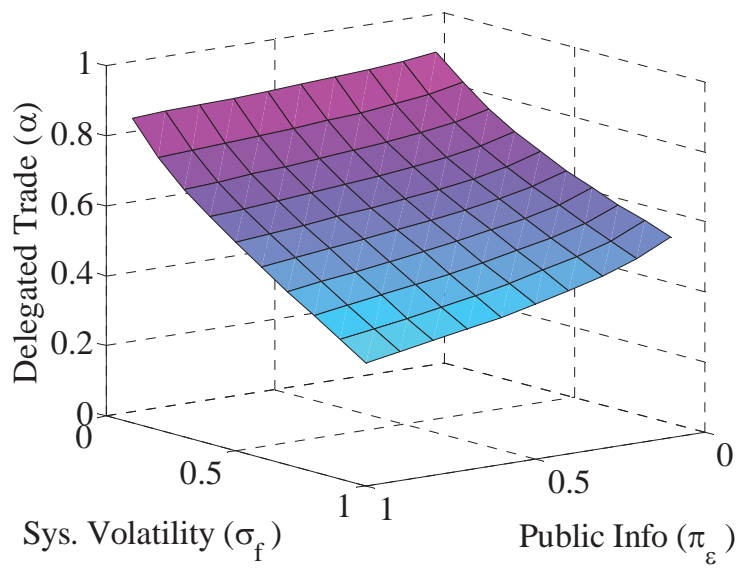

Panel B. Illiquidity

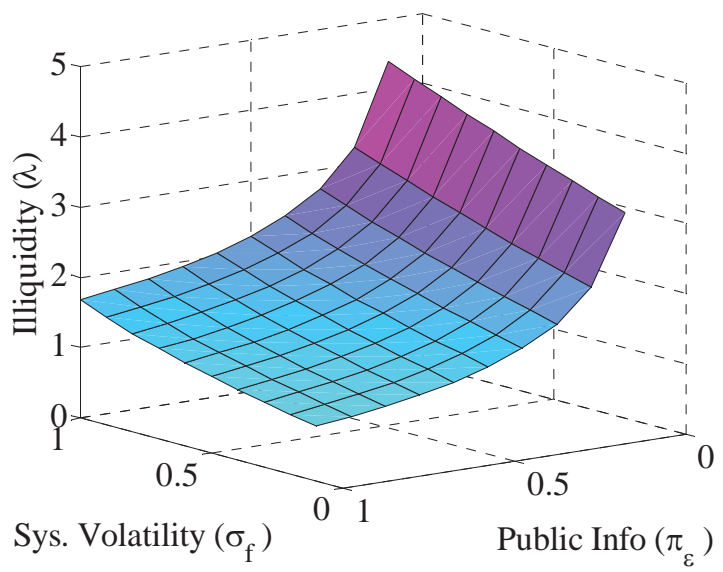

Panel C. Risk Premium

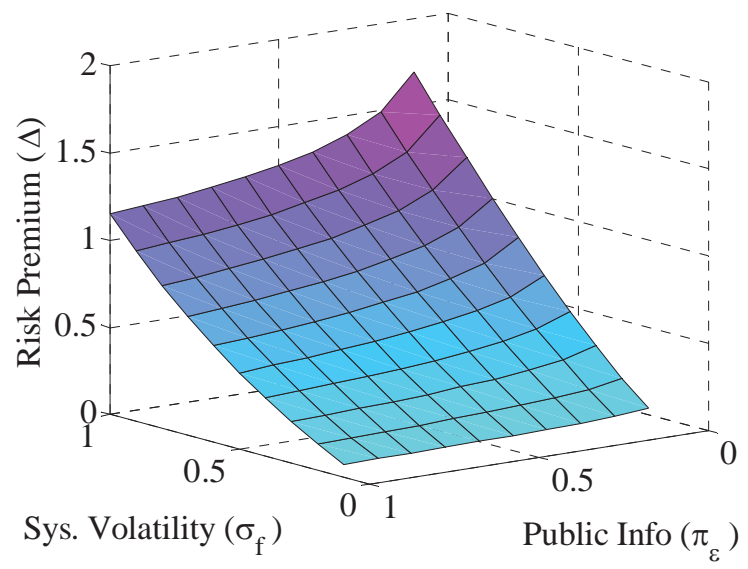

Panel A plots the relation among delegated trade $(\alpha)$, public information $\left(\pi_{\varepsilon}\right)$, and systematic cash flow volatility $\left(\sigma_{f}\right)$. Panel B plots the relation among illiquidity $(\lambda)$, public information $\left(\pi_{\varepsilon}\right)$, and systematic cash flow volatility $\left(\sigma_{f}\right)$. Panel $\mathrm{C}$ plots the relation among the risk premium $(\Delta)$, public information $\left(\pi_{\varepsilon}\right)$, and systematic cash flow volatility $\left(\sigma_{f}\right)$. In Panel A the $y$-axis is increasing bottom-to-top, the $x$-axis is increasing left-to-right, and the $z$-axis is increasing right-to-left. In Panels B and $\mathrm{C}$ the $y$-axis is increasing bottom-to-top, and the $x$ - and $z$-axes are increasing right-to-left. 
Figure 3. Effect of Public Information and Numerator of the Delegation Fee

Panel A. Delegated Trade

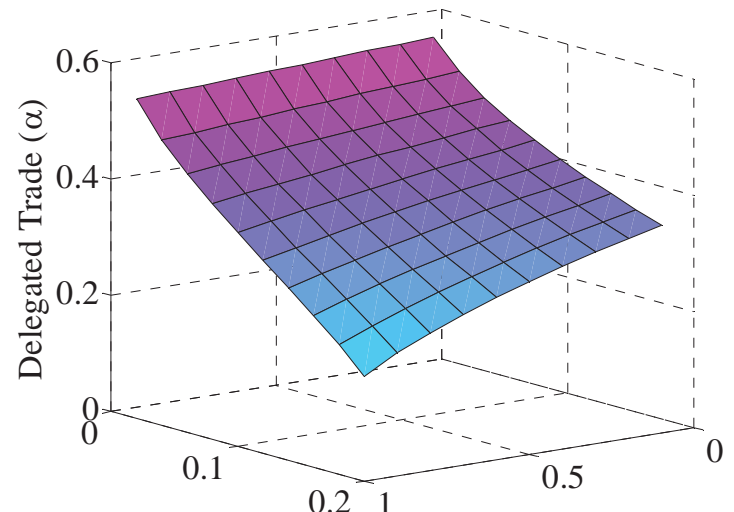

Num. Del. Fee $(\phi)$
Public Info $\left(\pi_{\varepsilon}\right)$
Panel B. Illiquidity

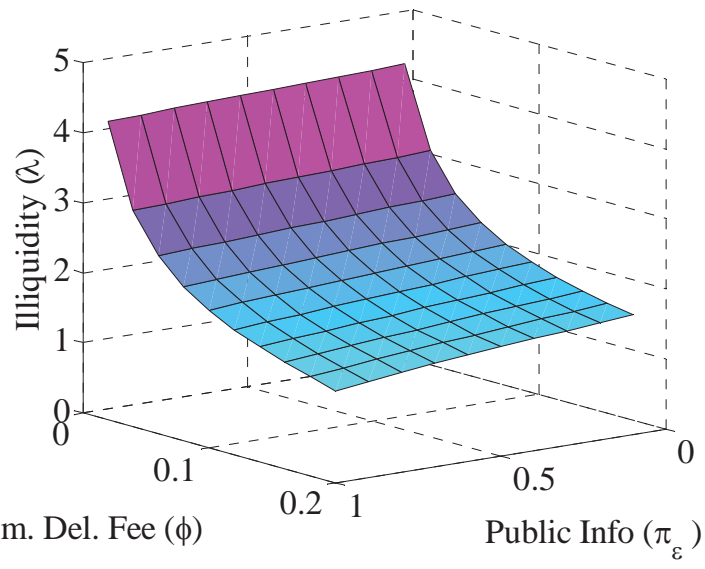

Panel C. Risk Premium

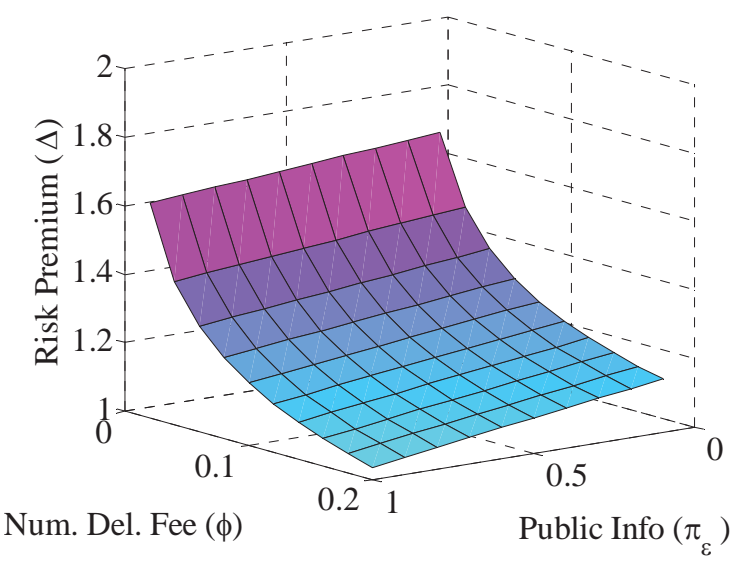

Panel A plots the relation among delegated trade $(\alpha)$, public information $\left(\pi_{\varepsilon}\right)$, and the numerator of the delegation fee $(\phi)$. Panel B plots the relation among illiquidity $(\lambda)$, public information $\left(\pi_{\varepsilon}\right)$, and the numerator of the delegation fee $(\phi)$. Panel C plots the relation among the risk premium $(\Delta)$, public information $\left(\pi_{\varepsilon}\right)$, and the numerator of the delegation fee $(\phi)$. In each panel, the $y$-axis is increasing bottom-to-top, and the $x$ - and $z$-axes are increasing right-to-left. 
Figure 4. Delegation Fee and Price Informativeness

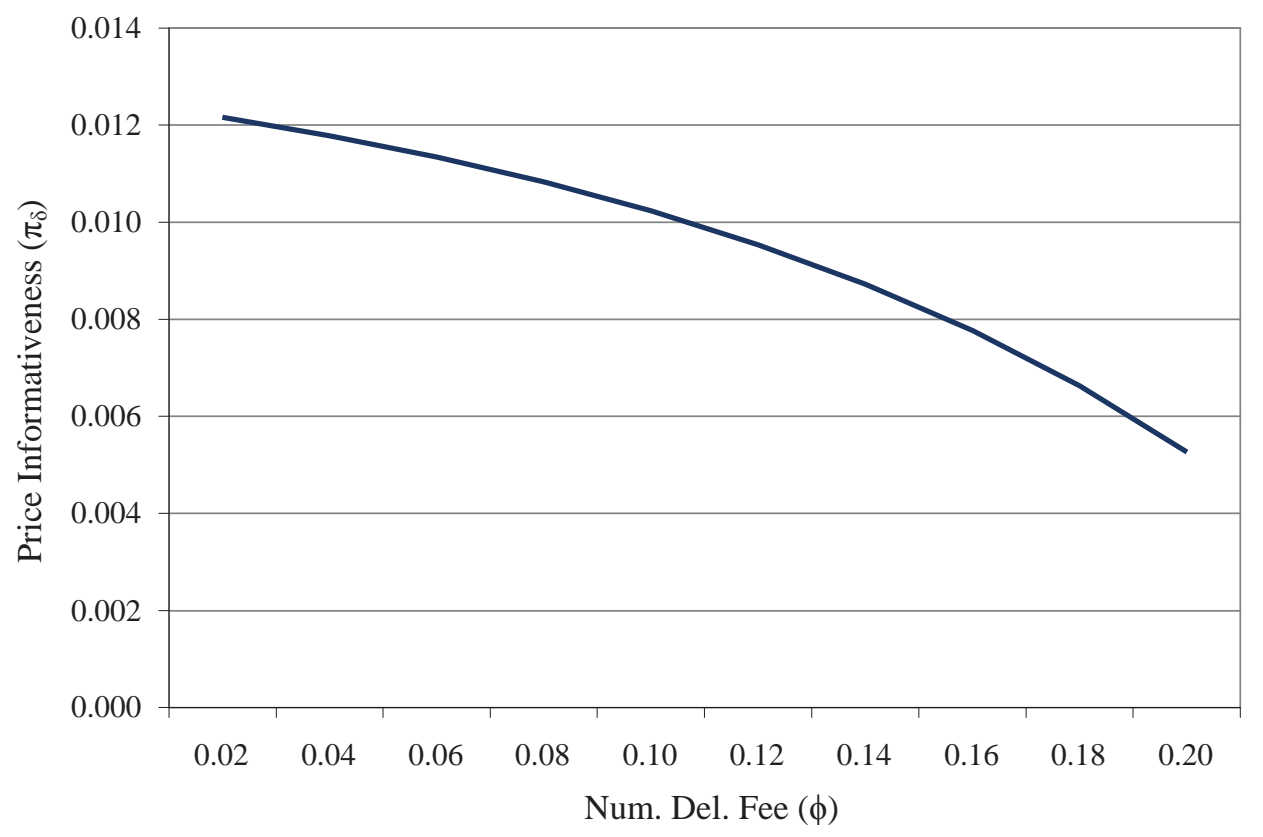

This figures plots the relation between the numerator of the delegation fee $(\phi)$ and the equilibrium level of price informativeness $\left(\pi_{\delta}\right)$. 


\section{Appendix A - Notation of Key Parameters}

$N$ - The number of investors in the economy

$\tau$ - An investor's tolerance for risk

$\tilde{P}_{a}$ - The price of shares of the risky asset

$\tilde{V}_{a}$ - The risky asset's cash flow

$\mu_{a}$ - The risky asset's expected cash flow

$\beta$ - The risky asset's factor-beta

$\tilde{F}$ - The common factor, where $\tilde{F}$ has variance $\sigma_{F}^{2}=N \sigma_{f}^{2}$

$\tilde{\varepsilon}$ - The idiosyncratic component of the risky asset's cash flow, where $\tilde{\varepsilon}$ has variance $\sigma_{\varepsilon}^{2}$

$\alpha$ - The fraction of investors who delegate their investment decisions to an intermediary

$\pi_{\varepsilon}+\pi_{\xi}$ - The (total) precision of the intermediary's information

$\pi_{\xi}$ - The precision of the intermediary's private information

$D_{I a}, D_{U a}$ - Informed and uninformed investors' demands for the risky asset, respectively

$\lambda$ - The extent to which total informed demand, $\alpha N D_{I a}$, affects price, $\tilde{P}_{a}$

$\tilde{x}$ - The supply of shares of the risky asset, where $\tilde{x}$ has variance $\pi_{x}$

$\tilde{q}$ - The information an uninformed investor infers from price

$\pi_{\varepsilon}+\pi_{\delta}$ - The (total) precision of the uninformed investor's information

$\pi_{\delta}$ - The precision of the information an uninformed investor infers from price

$\Delta(\lambda, \cdot)$ - The risky asset's risk premium

$\frac{\phi}{N}$ - The fee an investor pays to become informed

$M$ - The number of intermediaries in the economy

$k, K$ - An intermediary's variable and fixed costs, respectively 


\section{Appendix B - Results related to Section 3}

In the proofs that follow, let $\pi_{F}$ and $\pi_{f}$ represent the reciprocals of $\sigma_{F}^{2}$ and $\sigma_{f}^{2}$, respectively: as such, $\pi_{F}$ and $\pi_{f}$ represent the precisions (i.e., the reciprocal of variance) of each respective variable. We report and prove our results exclusively in terms of precision.

Proof to Proposition 1. To determine $\Lambda(\lambda, \cdot)$, we substitute in the expression for $\pi_{\delta}$ in eqn. (11) into eqn. (20) and then solve for $\lambda$. Ignoring proportionality factors, this yields the following expression for $\Lambda(\lambda, \cdot): \Lambda(\lambda, \cdot)=c_{3} \lambda^{3}+c_{2} \lambda^{2}-c_{1} \lambda-c_{0}$, where the coefficients $c_{j}$ are defined by

$$
\begin{aligned}
c_{3}= & \left(N \tau \pi_{F}\right)^{3} \pi_{\varepsilon}\left(\pi_{\varepsilon}+\pi_{\xi}\right)^{2} \alpha^{2}(1-\alpha), \\
c_{2}= & \left(N \tau \pi_{F}\right)^{2}\left(\pi_{\varepsilon}+\pi_{\xi}\right) \alpha\left(\pi_{\varepsilon}(2-3 \alpha)\left(\pi_{F}+\beta^{2}\left(\pi_{\varepsilon}+\pi_{\xi}\right)\right)-\alpha \pi_{F} \pi_{\xi}\right), \\
c_{1}= & N \tau \pi_{F}\left(\left(N \tau \pi_{F}\right)^{2} \pi_{\xi}\left(\pi_{\varepsilon}+\pi_{\xi}\right) \pi_{x} \alpha^{2}(1-\alpha)\right. \\
& \left.+\left(\pi_{F}+\beta^{2}\left(\pi_{\varepsilon}+\pi_{\xi}\right)\right)\left(\pi_{\varepsilon}(3 \alpha-1)\left(\pi_{F}+\beta^{2}\left(\pi_{\varepsilon}+\pi_{\xi}\right)\right)+2 \alpha \pi_{F} \pi_{\xi}\right)\right), \\
c_{0}= & \left(\pi_{F}+\beta^{2}\left(\pi_{\varepsilon}+\pi_{\xi}\right)\right) \\
& \times\left(\left(\pi_{F}+\beta^{2} \pi_{\varepsilon}\right)\left(\pi_{F}+\beta^{2}\left(\pi_{\varepsilon}+\pi_{\xi}\right)\right)+\left(N \tau \pi_{F}\right)^{2} \alpha \pi_{\xi} \pi_{x}\right) .
\end{aligned}
$$

Note that $c_{3}$ and $c_{0}$ are both positive, but the signs of $c_{2}$ and $c_{1}$ are indeterminate. Because $c_{3}$ and $c_{0}$ are both positive, there exists some $\lambda>0$ that solves $\Lambda(\lambda, \cdot)=0$. For $\Lambda(\lambda, \cdot)=0$ to have multiple, positive $\lambda$-roots, Descartes' rule of signs requires that $c_{2}$ and $c_{1}$ both be negative (when $c_{3}$ and $c_{0}$ are both positive). A necessary and sufficient condition that $c_{2}$ is negative is that $\alpha>\frac{2 \pi_{\varepsilon}\left(\pi_{F}+\beta^{2}\left(\pi_{\varepsilon}+\pi_{\xi}\right)\right)}{3 \pi_{\varepsilon}\left(\pi_{F}+\beta^{2}\left(\pi_{\varepsilon}+\pi_{\xi}\right)\right)+\pi_{F} \pi_{\xi}}$, and a necessary (but not sufficient) condition that $c_{1}$ is negative is that $\alpha<\frac{\pi_{\varepsilon}\left(\pi_{F}+\beta^{2}\left(\pi_{\varepsilon}+\pi_{\xi}\right)\right)}{3 \pi_{\varepsilon}\left(\pi_{F}+\beta^{2}\left(\pi_{\varepsilon}+\pi_{\xi}\right)\right)+2 \pi_{F} \pi_{\xi}}$. But there is no $\alpha$ that satisfies both necessary conditions, and so $c_{2}$ and $c_{1}$ cannot both be negative: this establishes the existence of a unique $\lambda>0$ that satisfies $\Lambda(\lambda, \cdot)=0$. Let $\lambda^{*}$ represent the equilibrium $\lambda$ : that is, $\Lambda\left(\lambda^{*}, \cdot\right)=0$. In addition, let $\frac{d}{d \lambda} \Lambda\left(\lambda^{*}, \cdot\right)$ represent the derivative of $\Lambda(\lambda, \cdot)$ evaluated 
at $\lambda^{*}$ : that is, $\frac{d}{d \lambda} \Lambda\left(\lambda^{*}, \cdot\right)=\left.\frac{d}{d \lambda} \Lambda(\lambda, \cdot)\right|_{\lambda=\lambda^{*}}$. In the proofs that follow, it will be useful to establish two features of the equilibrium. The first is that $\frac{d}{d \lambda} \Lambda\left(\lambda^{*}, \cdot\right)>0$. To establish this feature, note that $c_{2}$ and $c_{1}$ cannot both be negative. Thus, suppose $c_{2}$ is positive. Then $\frac{d}{d \lambda} \Lambda\left(\lambda^{*}, \cdot\right)=\frac{d}{d \lambda} \Lambda\left(\lambda^{*}, \cdot\right)-\frac{\Lambda\left(\lambda^{*}, \cdot\right)}{\lambda^{*}}=2 c_{3}\left(\lambda^{*}\right)^{2}+c_{2} \lambda^{*}+\left(\lambda^{*}\right)^{-1} c_{0}>0$ where the second equality results from the fact that $\Lambda\left(\lambda^{*}, \cdot\right)=0$ in equilibrium. Alternatively, suppose $c_{1}$ is positive. Then $\frac{d}{d \lambda} \Lambda\left(\lambda^{*}, \cdot\right)=\frac{d}{d \lambda} \Lambda\left(\lambda^{*}, \cdot\right)-2 \frac{\Lambda\left(\lambda^{*}, \cdot\right)}{\lambda^{*}}=c_{3}\left(\lambda^{*}\right)^{2}+c_{1} \lambda^{*}+2\left(\lambda^{*}\right)^{-1} c_{0}>0$. The second feature is that because $\Lambda(\lambda=0, \cdot)<0$ and $\frac{d}{d \lambda} \Lambda\left(\lambda^{*}, \cdot\right)>0$, any $\hat{\lambda}$, say, that yields $\Lambda(\hat{\lambda}, \cdot)<0$ must have the property that $\hat{\lambda} \in\left[0, \lambda^{*}\right)$ and thus $\hat{\lambda}<\lambda^{*}$. In effect, $\Lambda(\lambda, \cdot)$ starts out negative at $\lambda=0$, and crosses the $x$-axis only once at $\lambda^{*}$ - only once because $\frac{d}{d \lambda} \Lambda\left(\lambda^{*}, \cdot\right)>0$. For example, to cross the $x$-axis more than once would require some additional $\lambda^{* *}$, say, such that $\Lambda\left(\lambda^{* *}, \cdot\right)=0$ and $\frac{d}{d \lambda} \Lambda\left(\lambda^{* *}, \cdot\right)<0$, and this can never be the case. Thus, any $\hat{\lambda}$ that yields $\Lambda(\hat{\lambda}, \cdot)<0$ has the property that $\hat{\lambda}<\lambda^{*}$. Q.E.D.

Proof to Proposition 2. First, determine $\Lambda(\lambda, \cdot)$ when $\pi_{F}^{-1}=N \pi_{f}^{-1}$ as $N$ becomes large. To do this, substitute in the expression for $\pi_{\delta}$ in eqn. (11) into eqn. (20) and then solve for $\lambda$ as $N$ becomes large. Unlike the expression for $\Lambda(\lambda, \cdot)$ in Proposition 1 , however, here we do not ignore proportionality factors because these factors are a function of $N$. This yields $\Lambda(\lambda, \cdot)=c_{3} \lambda^{3}+c_{2} \lambda^{2}-c_{1} \lambda-c_{0}$, where the coefficients $c_{j}$ are defined by

$$
\begin{aligned}
& c_{3}=\alpha^{2}(1-\alpha) \pi_{\varepsilon}\left(\pi_{\varepsilon}+\pi_{\xi}\right)\left(\tau \pi_{f}\right)^{3}, \\
& c_{2}=\alpha(2-3 \alpha) \pi_{\varepsilon}\left(\pi_{\varepsilon}+\pi_{\xi}\right)\left(\tau \beta \pi_{f}\right)^{2}, \\
& c_{1}=\left((1-\alpha) \pi_{\xi} \pi_{x}\left(\alpha \tau \pi_{f}\right)^{2}+(3 \alpha-1) \pi_{\varepsilon}\left(\pi_{\varepsilon}+\pi_{\xi}\right) \beta^{4}\right) \tau \pi_{f}, \\
& c_{0}=\beta^{2}\left(\pi_{\varepsilon}\left(\pi_{\varepsilon}+\pi_{\xi}\right) \beta^{4}+\alpha\left(\tau \pi_{f}\right)^{2} \pi_{\xi} \pi_{x}\right) .
\end{aligned}
$$

Next determine $\Delta(\lambda, \cdot)$ when $\pi_{F}^{-1}=N \pi_{f}^{-1}$ as $N$ becomes large: this yields eqn. (23). To establish that the risk premium is positive, note that $\Delta(\lambda, \cdot)$ has two features. First, $\Delta(\lambda, \cdot)$ 
increases as $\lambda$ increases. Second, $\Delta(\lambda, \cdot)$ has a unique, positive $\lambda$-root: call this root $\hat{\lambda}$. Using the expression for $\Lambda(\lambda, \cdot)$ above, one can show that $\Lambda(\hat{\lambda}, \cdot)<0$, which implies $\hat{\lambda}<\lambda^{*}$ from the Proof to Proposition 1. Hence, $\Delta\left(\lambda^{*}, \cdot\right)>0$ because $\Delta(\lambda, \cdot)$ is increasing in $\lambda$. Q.E.D.

Proof to Corollary 1. Recall two facts from the Proof to Proposition 1. First, in equilibrium $\Lambda\left(\lambda^{*}, \cdot\right)=0$; second, $\frac{d}{d \lambda} \Lambda\left(\lambda^{*}, \cdot\right)>0$. Thus, using the expression for $\Lambda(\lambda, \cdot)$ in the Proof to Proposition 2, one can show:

$$
\begin{aligned}
\frac{d}{d \pi_{\xi}} \lambda^{*} & =-\frac{\frac{d}{d \pi_{\xi}} \Lambda\left(\lambda^{*}, \cdot\right)-\frac{\Lambda\left(\lambda^{*}, \cdot\right)}{\pi_{\varepsilon}+\pi_{\xi}}}{\frac{d}{d \lambda} \Lambda\left(\lambda^{*}, \cdot\right)}=\alpha \tau^{2} \pi_{f}^{2} \pi_{x} \frac{\pi_{\varepsilon}}{\pi_{\varepsilon}+\pi_{\xi}} \frac{\alpha \tau(1-\alpha) \pi_{f} \lambda^{*}+\beta^{2}}{\frac{d}{d \lambda} \Lambda\left(\lambda^{*}, \cdot\right)}>0 \\
\frac{d}{d \pi_{x}} \lambda^{*} & =-\frac{\frac{d}{d \pi_{x}} \Lambda\left(\lambda^{*}, \cdot\right)}{\frac{d}{d \lambda} \Lambda\left(\lambda^{*}, \cdot\right)}=\alpha \tau^{2} \pi_{f}^{2} \pi_{\xi} \frac{\alpha \tau(1-\alpha) \pi_{f} \lambda^{*}+\beta^{2}}{\frac{d}{d \lambda} \Lambda\left(\lambda^{*}, \cdot\right)}>0 \\
\frac{d}{d \pi_{\varepsilon}} \lambda^{*} & =-\frac{\frac{d}{d \pi_{\varepsilon}} \Lambda\left(\lambda^{*}, \cdot\right)-\frac{2 \pi_{\varepsilon}+\pi_{\xi}}{\pi_{\varepsilon}\left(\pi_{\varepsilon}+\pi_{\xi}\right)} \Lambda\left(\lambda^{*}, \cdot\right)}{\frac{d}{d \lambda} \Lambda\left(\lambda^{*}, \cdot\right)}=-\alpha \tau^{2} \pi_{f}^{2} \pi_{x} \pi_{\xi} \frac{2 \pi_{\varepsilon}+\pi_{\xi}}{\pi_{\varepsilon}\left(\pi_{\varepsilon}+\pi_{\xi}\right)} \frac{\alpha \tau(1-\alpha) \pi_{f} \lambda^{*}+\beta^{2}}{\frac{d}{d \lambda} \Lambda\left(\lambda^{*}, \cdot\right)}<0 .
\end{aligned}
$$

The proof that establishes that the sign of $\frac{d}{d \alpha} \lambda^{*}$ is positive is complex. The steps are as follows. First, define

$$
L\left(\lambda^{*}, \cdot\right)=-\frac{d \Lambda\left(\lambda^{*}, \cdot\right)}{d \alpha}+\frac{1}{\alpha} \frac{\alpha \tau \pi_{f}(2-3 \alpha) \lambda+\beta^{2}}{\alpha \tau \pi_{f}(1-\alpha) \lambda+\beta^{2}} \Lambda\left(\lambda^{*}, \cdot\right)
$$

Second, show: 1) that the denominator of $L(\lambda, \cdot)$ is always positive; and 2) the numerator is positive when $\lambda=\frac{\beta^{2}}{(1-\alpha) \tau \pi_{f}}$ and increasing in $\lambda$. Third, show that $\Lambda\left(\frac{\beta^{2}}{(1-\alpha) \tau \pi_{f}}, \cdot\right)<0$, which implies $\frac{\beta^{2}}{(1-\alpha) \tau \pi_{f}}<\lambda^{*}$ from the Proof to Proposition 1 ; thus, the numerator of $L(\lambda, \cdot)$ evaluated at $\lambda=\lambda^{*}$ is positive and hence $L\left(\lambda^{*}, \cdot\right)$ is positive. Finally, because in equilibrium $\Lambda\left(\lambda^{*}, \cdot\right)=0$

$$
\frac{d}{d \alpha} \lambda^{*}=\frac{-\frac{d}{d \alpha} \Lambda\left(\lambda^{*}, \cdot\right)}{\frac{d}{d \lambda} \Lambda\left(\lambda^{*}, \cdot\right)}=\frac{L\left(\lambda^{*}, \cdot\right)}{\frac{d}{d \lambda} \Lambda\left(\lambda^{*}, \cdot\right)}>0 .
$$

This proves that $\lambda^{*}$ increases as $\alpha$ increases. As for the fact that $\lambda^{*}$ can either increase or 
decrease as $\tau, \pi_{f}$ (where $\pi_{f}=\sigma_{f}^{-2}$ ), or $\beta$ increases, each of these parameters operates on $\Lambda(\lambda, \cdot)$ (as defined in the Proof to Proposition 2) in an equivalent way. For example, if one defines a parameter $s=\tau \beta^{-2} \pi_{f}$, then $\Lambda(\lambda, \cdot)$ reduces to an expression that is exclusively a function of $s$. Thus, it suffices to prove this claim for one of $\tau, \pi_{f}$, or $\beta$ : we prove it for $\tau$. First, let $\boldsymbol{\omega}_{1}=\left\{10,1,1,1,215.38, \frac{1}{10}, 1\right\}$ and $\boldsymbol{\omega}_{2}=\left\{1,1,1,1,127.69, \frac{2}{3}, 1\right\}$; one can show that $\Lambda\left(10, \boldsymbol{\omega}_{1}\right)=0$ and $\Lambda\left(10, \boldsymbol{\omega}_{2}\right)=0$. Next, show that

$$
\left.\frac{d}{d \tau} \Lambda(\lambda, \cdot)\right|_{\lambda=10, \omega_{1}}=-152.02 \text { and }\left.\frac{d}{d \tau} \Lambda(\lambda, \cdot)\right|_{\lambda=10, \omega_{2}}=131.2
$$

Thus, the sign of $\frac{d}{d \tau} \lambda^{*}=-\frac{\frac{d}{d \tau} \Lambda\left(\lambda^{*}, \cdot\right)}{\frac{d}{d \lambda} \Lambda\left(\lambda^{*}, \cdot\right)}$ can be either positive or negative. Q.E.D.

Proof to Corollary 2. We prove this for the effect of a change in $\pi_{\varepsilon}$ on the risk premium: the proof for $\pi_{\xi}$ is identical in spirit. The effect of a change in $\pi_{\varepsilon}$ on $\Delta\left(\lambda^{*}, \cdot\right)$ is:

$$
\begin{aligned}
& \frac{d}{d \pi_{\varepsilon}} \Delta\left(\lambda^{*}, \cdot\right)+\frac{d}{d \lambda} \Delta\left(\lambda^{*}, \cdot\right) \frac{d}{d \pi_{\varepsilon}} \lambda^{*} \\
= & \frac{\frac{d}{d \pi_{\varepsilon}} \Delta\left(\lambda^{*}, \cdot\right) \frac{d}{d \lambda} \Lambda\left(\lambda^{*}, \cdot\right)+\frac{d}{d \lambda} \Delta\left(\lambda^{*}, \cdot\right)\left(-\alpha \tau^{2} \pi_{f}^{2} \pi_{x} \pi_{\xi} \frac{2 \pi_{\varepsilon}+\pi_{\xi}}{\pi_{\varepsilon}\left(\pi_{\varepsilon}+\pi_{\xi}\right)}\left(\alpha \tau(1-\alpha) \pi_{f} \lambda^{*}+\beta^{2}\right)\right)}{\frac{d}{d \lambda} \Lambda\left(\lambda^{*}, \cdot\right)}(25)
\end{aligned}
$$

where in eqn. (25).we substitute in the expression for $\frac{d}{d \pi_{\varepsilon}} \lambda^{*}$ from Corollary 1. From the Proof to Proposition $1 \frac{d}{d \lambda} \Lambda\left(\lambda^{*}, \cdot\right)>0$, and thus the denominator of the expression in eqn. (25) is positive. Let $\Phi\left(\lambda^{*}, \cdot\right)$ represent the numerator of the expression (ignoring positive proportional terms). Then, recalling that $\Lambda\left(\lambda^{*}, \cdot\right)=0$, one can show that the numerator reduces to

$$
\Phi\left(\lambda^{*}, \cdot\right)-\left(\frac{4 \tau \alpha(1-\alpha) \lambda+\frac{\beta^{2}}{\pi_{f}}(3-\alpha)}{\pi_{f}^{2}\left(\tau \alpha(1-\alpha) \pi_{f} \lambda+\beta^{2}\right)}\right) \Lambda\left(\lambda^{*}, \cdot\right)=-\frac{\alpha \beta^{4} \pi_{\varepsilon}\left(\pi_{\varepsilon}+\pi_{\xi}\right)\left(2 \tau \alpha \pi_{f} \lambda+\beta^{2}\right)^{2}}{\pi_{f}^{3}\left(\tau \alpha(1-\alpha) \pi_{f} \lambda+\beta^{2}\right)},
$$

where the right-hand-side of eqn. (26) is clearly negative. This proves the claim. Q.E.D. 
Proof to Corollary 3. To show $\Delta\left(\lambda^{*}, \cdot\right) \geq\left(\tau \pi_{f}\right)^{-1} \beta^{2}$, define a function $G\left(\lambda, \tau, \pi_{\varepsilon}, \pi_{\xi}, \pi_{f}, \pi_{x}, \alpha, \beta\right)=$ $\Delta(\lambda, \cdot)-\left(\tau \pi_{f}\right)^{-1} \beta^{2}$, where $\Delta(\lambda, \cdot)$ is defined as in eqn. (23). The function $G(\lambda, \cdot)$ has two features. It increases as $\lambda$ increases, and it has a unique, positive $\lambda$-root: call this root $\hat{\lambda}$. It is a tedious, but otherwise straightforward, exercise to show that $\Lambda(\hat{\lambda}, \cdot)<0$, where $\Lambda(\lambda, \cdot)$ is as defined in the Proof to Proposition 2. Because $\Lambda(\hat{\lambda}, \cdot)<0$, from the Proof to Proposition 1 it must be the case that the $\hat{\lambda}<\lambda^{*}$. But this, in turn, implies that $G\left(\lambda^{*}, \cdot\right)>0$ because $G(\lambda, \cdot)$ increases as $\lambda$ increases, and hence $\Delta\left(\lambda^{*}, \cdot\right) \geq\left(\tau \pi_{f}\right)^{-1} \beta^{2}$. Q.E.D.

\section{Appendix C - Results related to Section 4}

Proof to Lemma 1. Lemma 1 is very complex in detail, and so we attempt to reduce it to a series of smaller steps. In the first step, we determine the expected utilities of informed and uninformed investors. In the economy we posit, there are two (independent) random elements, or variables: $\tilde{y}$, informed investors' private information, and $\tilde{x}$, random supply. The former has mean 0 and precision $\pi_{y}=\left(\pi_{\varepsilon}^{-1}+\pi_{\xi}^{-1}\right)^{-1}$, and the latter has mean 1 and precision $\pi_{x}$. To start, an informed investor's ex ante expected utility is

$$
-E_{\tilde{y}, \tilde{x}}\left[E\left[\exp \left[-\frac{1}{\tau} D_{I a}\left(\tilde{V}_{a}-P_{a}\right)\right] \mid y, x\right]\right] .
$$

Substituting in the expressions for $D_{I a}$ in eqn. (7) and $P_{a}$ in eqn. (4) and simplifying allows eqn. (27) to be characterized as

$$
-E_{\tilde{y}, \tilde{x}}\left[E\left[\exp \left[-\frac{1}{\tau} D_{I a}\left(\tilde{V}_{a}-P_{a}\right)\right] \mid y, x\right]\right]=-E_{\tilde{y}, \tilde{x}}\left[\exp \left[-\frac{1}{2}\left(a_{I} \tilde{y}+b_{I} \tilde{x}+c_{I}\right)^{2}\right]\right],
$$


where

$$
\begin{aligned}
a_{I} & =\frac{\frac{\pi_{\xi}}{\pi_{\varepsilon}+\pi_{\xi}}}{\sqrt{N} \sqrt{\beta^{2} \sigma_{f}^{2}+N^{-1}\left(\pi_{\varepsilon}+\pi_{\xi}\right)^{-1}+2 \alpha \lambda \tau}}, \\
b_{I} & =\frac{\lambda}{\sqrt{N} \sqrt{\beta^{2} \sigma_{f}^{2}+N^{-1}\left(\pi_{\varepsilon}+\pi_{\xi}\right)^{-1}+2 \alpha \lambda \tau}}, \\
c_{I} & =-\frac{\theta}{\sqrt{N} \sqrt{\beta^{2} \sigma_{f}^{2}+N^{-1}\left(\pi_{\varepsilon}+\pi_{\xi}\right)^{-1}+2 \alpha \lambda \tau}} .
\end{aligned}
$$

Next determine the expression for an informed investor's expected utility that results from integrating over $\tilde{y}$ and $\tilde{x}$ in eqn. (28). Here the 4 key steps are:

$$
\begin{aligned}
& -E_{\tilde{y}, \tilde{x}}\left[\exp \left[-\frac{1}{2}\left(a_{I} \tilde{y}+b_{I} \tilde{x}+c_{I}\right)^{2}\right]\right] \\
& =-\frac{\sqrt{\pi_{y} \pi_{x}}}{2 \pi} \int_{y} \int_{x} \exp \left[-\frac{1}{2}\left(a_{I} y+b_{I} x+c_{I}\right)^{2}\right. \\
& \left.-\frac{1}{2}(y, \quad x-1)\left(\begin{array}{cc}
\pi_{y} & 0 \\
0 & \pi_{x}
\end{array}\right)\left(\begin{array}{c}
y \\
x-1
\end{array}\right)\right] d x d y \\
& =-\frac{\sqrt{\pi_{y} \pi_{x}}}{2 \pi} \int_{y} \int_{x} \exp \left[-\left(b_{I}+c_{I}\right)\left(a_{I} y+b_{I} x\right)-\frac{1}{2}\left(c_{I}^{2}-b_{I}^{2}\right)\right. \\
& \left.-\frac{1}{2}\left(\begin{array}{cc}
y, & x-1
\end{array}\right)\left(\begin{array}{cc}
\pi_{y}+a_{I}^{2} & a_{I} b_{I} \\
a_{I} b_{I} & \pi_{x}+b_{I}^{2}
\end{array}\right)\left(\begin{array}{c}
y \\
x-1
\end{array}\right)\right] d x d y \\
& =-\sqrt{\frac{\pi_{y} \pi_{x}}{\left|\left(\begin{array}{cc}
\pi_{y}+a_{I}^{2} & a_{I} b_{I} \\
a_{I} b_{I} & \pi_{x}+b_{I}^{2}
\end{array}\right)\right|}} \exp \left[-\left(b_{I}+c_{I}\right) b_{I}-\frac{1}{2}\left(c_{I}^{2}-b_{I}^{2}\right)\right. \\
& \left.+\frac{1}{2}\left(b_{I}+c_{I}\right)^{2}\left(a_{I}, \quad b_{I}\right)\left(\begin{array}{cc}
\pi_{y}+a_{I}^{2} & a_{I} b_{I} \\
a_{I} b_{I} & \pi_{x}+b_{I}^{2}
\end{array}\right)^{-1}\left(\begin{array}{c}
a_{I} \\
b_{I}
\end{array}\right)\right] \\
& =-\sqrt{\frac{\pi_{y} \pi_{x}}{\pi_{x} a_{I}^{2}+\pi_{y} b_{I}^{2}+\pi_{x} \pi_{y}}} \exp \left[-\frac{1}{2} \frac{\pi_{x} \pi_{y}\left(b_{I}+c_{I}\right)^{2}}{\pi_{x} a_{I}^{2}+\pi_{y} b_{I}^{2}+\pi_{x} \pi_{y}}\right] \text {. }
\end{aligned}
$$


Next, an uninformed investor's ex ante expected utility is

$$
-E_{\tilde{y}, \tilde{x}}\left[E\left[\exp \left[-\frac{1}{\tau} D_{U a}\left(\tilde{V}_{a}-P_{a}\right)\right] \mid q\right]\right] .
$$

Substituting in the expressions for $D_{U a}$ in eqn. (16) and $\tilde{P}_{a}$ in eqn. (4) and simplifying allows eqn. (34) to be characterized as

$$
-E_{\tilde{y}, \tilde{x}}\left[E\left[\exp \left[-\frac{1}{\tau} D_{U a}\left(\tilde{V}_{a}-P_{a}\right)\right] \mid q\right]\right]=-E_{\tilde{y}, \tilde{x}}\left[\exp \left[-\frac{1}{2}\left(a_{U} \tilde{y}+b_{U} \tilde{x}+c_{U}\right)^{2}\right]\right],
$$

where

$$
\begin{aligned}
a_{U}= & \frac{\frac{\pi_{\delta}}{\pi_{\varepsilon}+\pi_{\delta}}-\frac{\pi_{\xi}}{\pi_{\varepsilon}+\pi_{\xi}} \frac{\alpha \lambda \tau}{\beta^{2} \sigma_{f}^{2}+N^{-1}\left(\pi_{\varepsilon}+\pi_{\xi}\right)^{-1}+2 \alpha \lambda \tau}}{\sqrt{N} \sqrt{\beta^{2} \sigma_{f}^{2}+N^{-1}\left(\pi_{\varepsilon}+\pi_{\delta}\right)^{-1}}}, \\
b_{U}= & \frac{\lambda-\frac{\pi_{\delta}}{\pi_{\varepsilon}+\pi_{\delta}} \frac{\pi_{\varepsilon}+\pi_{\xi}}{\pi_{\xi}}\left(\lambda+\frac{\beta^{2} \sigma_{f}^{2}+N^{-1}\left(\pi_{\varepsilon}+\pi_{\xi}\right)^{-1}}{\alpha \tau}\right)-\frac{\alpha \lambda \tau}{\beta^{2} \sigma_{f}^{2}+N^{-1}\left(\pi_{\varepsilon}+\pi_{\xi}\right)^{-1}+2 \alpha \lambda \tau}}{\sqrt{N} \sqrt{\beta^{2} \sigma_{f}^{2}+N^{-1}\left(\pi_{\varepsilon}+\pi_{\delta}\right)^{-1}}}, \\
c_{U}= & \frac{\frac{\alpha \lambda \tau}{\beta^{2} \sigma_{f}^{2}+N^{-1}\left(\pi_{\varepsilon}+\pi_{\xi}\right)^{-1}+2 \alpha \lambda \tau}-\frac{\pi_{\delta}}{\pi_{\varepsilon}+\pi_{\delta}} \frac{\pi_{\varepsilon}+\pi_{\xi}}{\pi_{\xi}}}{\sqrt{N} \sqrt{\beta^{2} \sigma_{f}^{2}+N^{-1}\left(\pi_{\varepsilon}+\pi_{\delta}\right)^{-1}}} \theta .
\end{aligned}
$$

Repeating the analysis above in eqns. (29)-(33), but with $a_{U}, b_{U}$, and $c_{U}$ in place of $a_{I}, b_{I}$, and $c_{I}$, yields the following expression for an uninformed investor's expected utility

$$
-\sqrt{\frac{\pi_{y} \pi_{x}}{\pi_{x} a_{U}^{2}+\pi_{y} b_{U}^{2}+\pi_{x} \pi_{y}}} \exp \left[-\frac{1}{2} \frac{\pi_{x} \pi_{y}\left(b_{U}+c_{U}\right)^{2}}{\pi_{x} a_{U}^{2}+\pi_{y} b_{U}^{2}+\pi_{x} \pi_{y}}\right] .
$$

The second step is to assume that each informed investor pays a per-informed-investor fee of $\frac{\phi}{N}$, and then compute the ratio of an informed investor's expected utility to an uninformed investor's expected utility: in effect, the ratio of eqn. (33) to eqn. (35). For the moment, let us refer to this ratio as "Ratio." As in Grossman and Stiglitz (1980), showing that for 
any $\frac{\phi}{N}$ there exists an $\alpha^{*}$, say, such that Ratio is 1 is tantamount to establishing that there exists an $\alpha^{*}$-fraction of informed investors versus a $\left(1-\alpha^{*}\right)$-fraction of uninformed investors such that an investor is indifferent between paying the fee and becoming informed versus remaining uninformed. This, in turn, is equivalent to computing

$$
\lim _{N \rightarrow \infty} N \cdot \ln [\text { Ratio }]
$$

and then showing that for any $\frac{\phi}{N}$ there exists an $\alpha^{*}$ such that $\lim _{N \rightarrow \infty} N \cdot \ln [$ Ratio $]=0$. Thus, our next step is to compute $\lim _{N \rightarrow \infty} N \cdot \ln [$ Ratio $]$; this calculation yields

$$
\lim _{N \rightarrow \infty} N \cdot \ln [\text { Ratio }]=\Omega\left(\lambda, \alpha, \beta, \tau, \sigma_{f}, \pi_{\varepsilon}, \pi_{\xi}, \pi_{x}\right)+2 \frac{\phi}{\tau},
$$

where $\Omega(\cdot)$ can be expressed as the ratio of two $8^{\text {th }}$-order polynomials in $\alpha$, where the coefficients to the $\alpha$-polynomials are functions of $\lambda, \beta, \tau, \sigma_{f}, \pi_{\varepsilon}, \pi_{\xi}$, and $\pi_{x}$. Suffice it to say that $\Omega(\cdot)$ is a very complicated expression (we spare the reader the details). Exacerbating the complication, however, is the fact that $\Omega(\cdot)$, in addition to being a function of a host of exogenous parameters, is also a function of $\lambda$ and $\lambda$ is an endogenous variable. For example, from the proof to Proposition 2 in Appendix B, we know that $\lambda$ has to satisfy $\Lambda(\lambda, \cdot)=c_{3} \lambda^{3}+c_{2} \lambda^{2}-c_{1} \lambda-c_{0}$, where the coefficients $c_{j}$ are defined by

$$
\begin{aligned}
& c_{3}=\alpha^{2}(1-\alpha) \pi_{\varepsilon}\left(\pi_{\varepsilon}+\pi_{\xi}\right)\left(\tau \pi_{f}\right)^{3}, \\
& c_{2}=\alpha(2-3 \alpha) \pi_{\varepsilon}\left(\pi_{\varepsilon}+\pi_{\xi}\right)\left(\tau \beta \pi_{f}\right)^{2}, \\
& c_{1}=\left((1-\alpha) \pi_{\xi} \pi_{x}\left(\alpha \tau \pi_{f}\right)^{2}+(3 \alpha-1) \pi_{\varepsilon}\left(\pi_{\varepsilon}+\pi_{\xi}\right) \beta^{4}\right) \tau \pi_{f}, \\
& c_{0}=\beta^{2}\left(\pi_{\varepsilon}\left(\pi_{\varepsilon}+\pi_{\xi}\right) \beta^{4}+\alpha\left(\tau \pi_{f}\right)^{2} \pi_{\xi} \pi_{x}\right),
\end{aligned}
$$

and $\pi_{f}=\sigma_{f}^{-2}$. Because the solution to $\lambda$ is a $3^{r d}$-order polynomial, solving for $\alpha$ and $\lambda$ 
simultaneously results in $\Omega(\cdot)$ implicitly being the ratio of two $11^{\text {th }}$-order polynomials in $\alpha$, where the coefficients to the $\alpha$-polynomials are functions of $\beta, \tau, \sigma_{f}, \pi_{\varepsilon}, \pi_{\xi}$, and $\pi_{x}$ (all of which are exogenous parameters). Despite the level of complexity, it is straightforward to establish two facts: 1) $\alpha=0$ implies $\lambda=\tau^{-1} \beta^{2} \sigma_{f}^{2}$ (where $\sigma_{f}^{2}=\pi_{f}^{-1}$ ); and 2) $\alpha \rightarrow 1$ implies $\lambda \rightarrow \infty$. These two facts, in turn, imply

$$
\Omega\left(\lambda=\tau^{-1} \beta^{2} \sigma_{f}^{2}, \alpha=0, \beta, \tau, \sigma_{f}, \pi_{\varepsilon}, \pi_{\xi}, \pi_{x}\right)=-\frac{\pi_{\xi}}{\pi_{x}} \frac{\pi_{\xi}+\pi_{x}}{\left(\pi_{\varepsilon}+\pi_{\xi}\right)^{2}} \frac{1}{\beta^{2} \sigma_{f}^{2}},
$$

and

$$
\lim _{\alpha \rightarrow 1} \lim _{\lambda \rightarrow \infty} \Omega\left(\lambda, \alpha, \beta, \tau, \sigma_{f}, \pi_{\varepsilon}, \pi_{\xi}, \pi_{x}\right) \rightarrow+\infty
$$

To summarize the discussion to this point, we have established that $\Omega(\cdot)$, as a function of $\alpha$, has a range between $-\frac{\pi_{\xi}}{\pi_{x}} \frac{\pi_{x}+\pi_{\xi}}{\left(\pi_{\varepsilon}+\pi_{\xi}\right)^{2}} \frac{1}{\beta^{2} \sigma_{f}^{2}}$ and $+\infty$, and, as a ratio of polynomials in $\alpha$, is continuous and (mathematically) well-behaved. Collectively, this implies from the intermediate value theorem that for any set of exogenous parameters $\beta, \tau, \sigma_{f}, \pi_{\varepsilon}, \pi_{\xi}, \pi_{x}$, and $\phi \in\left[0, \frac{\tau}{2} \frac{\pi_{\xi}}{\pi_{x}} \frac{\pi_{x}+\pi_{\xi}}{\left(\pi_{\varepsilon}+\pi_{\xi}\right)^{2}} \frac{1}{\beta^{2} \sigma_{f}^{2}}\right]$, there exists some $\alpha^{*}$, say, such that

$$
\Omega\left(\lambda, \alpha^{*}, \beta, \tau, \sigma_{f}, \pi_{\varepsilon}, \pi_{\xi}, \pi_{x}\right)+2 \frac{\phi}{\tau}=0
$$

This proves the claim in the statement of Lemma 1. Q.E.D.

An implication of $\alpha=0$. At first blush it may seem odd that that $\alpha=0$ implies $\lambda=\tau^{-1} \beta^{2} \sigma_{f}^{2}$ because if $\alpha=0$ then there are no informed investors, and thus in the absence of informed trade one might expect the market to be perfectly liquid (i.e., $\lambda=0$ ). But recall that investors' conjecture about the price of the risky asset is that it is of the form $P_{a}=\mu_{a}+\theta+\lambda\left(\alpha N \cdot \tilde{D}_{I a}-\tilde{x}\right)$, and so $\alpha=0$ implies that market illiquidity arises as a consequence of (exclusively) random supply, $\tilde{x}$. In other words, random supply behaves like a 
large, uninformed investor who, despite being uninformed, nonetheless makes markets illiquid through the impact of his demand on price. Here it may be useful to think of the random supply $(\tilde{x})$ as a large, uninformed block trade. This points to the fact that information, per se, is not a requirement for illiquidity: large demand orders, such as large block trades, are sufficient to make markets illiquid. Thus, in equilibrium $\lambda=\tau^{-1} \beta^{2} \sigma_{f}^{2}$. Nonetheless, when $\alpha=0$ (i.e., in the absence of informed trade), the expression for the risk premium in eqn. (23) reduces to the same expression for the risk premium as when the market is perfectly competitive $\left(\tau^{-1} \beta^{2} \sigma_{f}^{2}\right)$ : in other words, $\Delta\left(\lambda=\tau^{-1} \beta^{2} \sigma_{f}^{2}, \ldots, \alpha=0, \ldots\right)=\tau^{-1} \beta^{2} \sigma_{f}^{2}$.

\section{Appendix D - Results related to Section 5}

In Appendix D we prove Proposition 4. As there is no private information about the common factor, we assume that all investors act as price takers in their demands for the traded common factor. As in the previous section, uninformed investors act as price takers in their demands for the risky asset whereas an intermediary coordinates the demands of informed investors. Let $D_{t T}$ and $D_{t a}$ represent investors' percentage demands for the common factor and the risky asset, respectively, where $t \in[I, U]$ represents an investor's type, either informed $(I)$ or uninformed $(U)$. Finally, recall that we represent the price of the risky asset as $P_{a}$, where the intermediary conjectures that $P_{a}=\mu_{a}+\theta+\lambda\left(\alpha N D_{I a}-x\right)$. Henceforth we represent the price of the common factor as $P_{T}$.

The first step is to determine the $1^{\text {st }}$-order conditions that characterize informed and uninformed investors' demand for the risky asset and the common factor. In conjunction with this, we prove the following lemma.

Lemma 2. The following equations characterize the $1^{\text {st }}$-order conditions that result from the intermediary coordinating informed trade conditional on knowledge of $\tilde{y}=y$ and $\tilde{x}=x$, and an uninformed investor solving his optimization problem. For an informed investor, the 
$1^{\text {st }}$-order conditions are

$$
\begin{aligned}
& 0=\mu-P_{T}-\frac{1}{\tau}\left(\left(\sigma_{F}^{2}+\sigma_{T}^{2}\right) D_{I T}+\beta \sigma_{F}^{2} D_{I a}\right) \\
& 0=E[\tilde{\varepsilon} \mid y]-\theta-2 \lambda \alpha N D_{I a}+\lambda x-\frac{1}{\tau}\left(\beta \sigma_{F}^{2} D_{I T}+\left(\beta^{2} \sigma_{F}^{2}+\operatorname{Var}[\tilde{\varepsilon} \mid y]\right) D_{I a}\right) ;
\end{aligned}
$$

and for an uninformed investor, the $1^{\text {st }}$-order conditions are

$$
\begin{aligned}
& 0=\mu-P_{T}-\frac{1}{\tau}\left(\left(\sigma_{F}^{2}+\sigma_{T}^{2}\right) D_{U T}+\beta \sigma_{F}^{2} D_{U a}\right) \\
& 0=\mu_{a}+E[\tilde{\varepsilon} \mid q]-P_{a}-\frac{1}{\tau}\left(\beta \sigma_{F}^{2} D_{U T}+\left(\beta^{2} \sigma_{F}^{2}+\operatorname{Var}[\tilde{\varepsilon} \mid q]\right) D_{U a}\right) .
\end{aligned}
$$

Proof to Lemma 2. Let $\mathbf{D}_{I}$ represent the 1 -by-2 vector $\left(D_{I T}, D_{I a}\right)$, and $\mathbf{D}_{I}^{T}$ its transpose. With this specification of the economy, the intermediary solves the following optimization problem for an informed investor conditional on knowledge of $\tilde{y}=y$ and $\tilde{x}=x$ :

$$
\begin{aligned}
& \max _{D_{I T}, D_{I a}} E_{\tilde{\mu}_{T}}\left[E_{\tilde{V}_{T}, \tilde{V}_{a}}\left[-\exp \left[-\frac{1}{\tau} \mathbf{D}_{I}\left(\begin{array}{c}
\tilde{V}_{T}-P_{T} \\
\tilde{V}_{a}-P_{a}
\end{array}\right)\right] \mid y, x\right]\right] \\
& =\max _{D_{I T}, D_{I a}} E_{\tilde{\mu}_{T}}\left[-\exp \left[\begin{array}{c}
-\frac{1}{\tau} \mathbf{D}_{I}\left(\begin{array}{c}
\tilde{\mu}_{T}-P_{T} \\
E[\tilde{\varepsilon} \mid y]-\theta-\lambda \alpha N D_{I a}+\lambda x
\end{array}\right) \\
+\frac{1}{2} \frac{1}{\tau^{2}} \mathbf{D}_{I}\left(\begin{array}{cc}
\sigma_{F}^{2} & \beta \sigma_{F}^{2} \\
\beta \sigma_{F}^{2} & \beta^{2} \sigma_{F}^{2}+\operatorname{Var}[\tilde{\varepsilon} \mid y]
\end{array}\right) \mathbf{D}_{I}^{T}
\end{array}\right]\right] \\
& =\max _{D_{I T}, D_{I a}}\left\{-\exp \left[\begin{array}{c}
-\frac{1}{\tau}\left(D_{I T}\left(\mu-P_{T}\right)+D_{I a}\left(E[\tilde{\varepsilon} \mid y]-\theta-\lambda \alpha N D_{I a}+\lambda x\right)\right)+\frac{1}{2 \tau^{2}} D_{I T}^{2} \sigma_{T}^{2} \\
+\frac{1}{2} \frac{1}{\tau^{2}}\left(D_{I T}^{2} \sigma_{F}^{2}+2 D_{I T} D_{I a} \beta \sigma_{F}^{2}+\left(\beta^{2} \sigma_{F}^{2}+\operatorname{Var}[\tilde{\varepsilon} \mid y]\right) D_{I a}^{2}\right)
\end{array}\right]\right\} .
\end{aligned}
$$


This yields the following $1^{\text {st }}$-order conditions for informed investors:

$$
\begin{aligned}
& 0=\mu-P_{T}-\frac{1}{\tau}\left(\left(\sigma_{F}^{2}+\sigma_{T}^{2}\right) D_{I T}+\beta \sigma_{F}^{2} D_{I a}\right) \\
& 0=E[\tilde{\varepsilon} \mid y]-\theta-2 \lambda \alpha N D_{I a}+\lambda x-\frac{1}{\tau}\left(\beta \sigma_{F}^{2} D_{I T}+\left(\beta^{2} \sigma_{F}^{2}+\operatorname{Var}[\tilde{\varepsilon} \mid y]\right) D_{I a}\right) .
\end{aligned}
$$

Let $\mathbf{D}_{U}$ represent the 1-by-2 vector $\left(D_{U T}, D_{U a}\right)$, and $\mathbf{D}_{U}^{T}$ its transpose. Uninformed investors condition their beliefs on $P_{T}$ and $P_{a}$ to infer information about $\tilde{\varepsilon}$ : let the statistic $\tilde{q}=q$ represent the information inferred. ${ }^{19}$ Uninformed investors solve the following optimization problem:

$$
\begin{aligned}
& \max _{D_{U T}, D_{U a}} E_{\tilde{\mu}_{T}}\left[E_{\tilde{V}_{T}, \tilde{V}_{a}}\left[-\exp \left[-\frac{1}{\tau} \mathbf{D}_{U}\left(\begin{array}{c}
\tilde{V}_{T}-P_{T} \\
\tilde{V}_{a}-P_{a}
\end{array}\right)\right] \mid q\right]\right] \\
& =\max _{D_{U T}, D_{U a}} E_{\tilde{\mu}_{T}}\left[-\exp \left[\begin{array}{c}
-\frac{1}{\tau} \mathbf{D}_{U}\left(\begin{array}{c}
\tilde{\mu}_{T}-P_{T} \\
\mu_{a}+E[\tilde{\varepsilon} \mid q]-P_{a}
\end{array}\right) \\
\left.+\frac{1}{2} \frac{1}{\tau^{2}} \mathbf{D}_{U}\left(\begin{array}{cc}
\sigma_{F}^{2} & \beta \sigma_{F}^{2} \\
\beta \sigma_{F}^{2} & \beta^{2} \sigma_{F}^{2}+\operatorname{Var}[\tilde{\varepsilon} \mid q]
\end{array}\right) \mathbf{D}_{U}^{T}\right]
\end{array}\right]\right. \\
& =\max _{D_{U T}, D_{U a}}\left\{-\exp \left[\begin{array}{c}
-\frac{1}{\tau}\left(D_{U T}\left(\mu-P_{T}\right)+D_{U a}\left(\mu_{a}+E[\tilde{\varepsilon} \mid q]-P_{a}\right)\right)+\frac{1}{2 \tau^{2}} D_{U T}^{2} \sigma_{T}^{2} \\
+\frac{1}{2} \frac{1}{\tau^{2}}\left(D_{U T}^{2} \sigma_{F}^{2}+2 D_{U T} D_{U a} \beta \sigma_{F}^{2}+\left(\beta^{2} \sigma_{F}^{2}+\operatorname{Var}[\tilde{\varepsilon} \mid q]\right) D_{U a}^{2}\right)
\end{array}\right]\right\} .
\end{aligned}
$$

This yields the following $1^{\text {st }}$-order conditions for uninformed investors:

$$
\begin{aligned}
0 & =\mu-P_{T}-\frac{1}{\tau}\left(\left(\sigma_{F}^{2}+\sigma_{T}^{2}\right) D_{U T}+\beta \sigma_{F}^{2} D_{U a}\right) \\
0 & =\mu_{a}+E[\tilde{\varepsilon} \mid q]-P_{a}-\frac{1}{\tau}\left(\beta \sigma_{F}^{2} D_{U T}+\left(\beta^{2} \sigma_{F}^{2}+\operatorname{Var}[\tilde{\varepsilon} \mid q]\right) D_{U a}\right)
\end{aligned}
$$

\section{Q.E.D.}

\footnotetext{
${ }^{19}$ Here, $\tilde{q}$ could be a vector of statistics (one each from $P_{M}$ and $P_{a}$ ) or a scalar if $P_{M}$ is not an additional source of information about $\tilde{\varepsilon}$.
} 
The next step is to implement these $1^{\text {st }}$-order conditions to determine market-clearing prices. Recall that $\tilde{x}$ represents the supply of the risky asset available for trade among informed and uninformed investors. Allowing investors to diversify into the common factor as a separate asset raises the possibility that $P_{T}$ will depend on $\tilde{x}$, and in this circumstance uninformed investors can use $P_{T}$ to improve their inferences about $\tilde{\varepsilon}$ from $P_{a}$. This complicates the problem considerably without having any qualitative effect on the results we report below. The reason it has no qualitative effect is that the key insight of our analysis is that only risk associated with the systematic component of the risky asset's cash flow manifests in the risk premium when investors can trade the common factor, and this insight is unrelated to uninformed investors' level of informedness - and hence unrelated to whether uninformed investors infer additional information from $P_{T}$. Thus, to facilitate the discussion, here we assume that the supply of the common factor available for trade is $-\frac{\sigma_{F}^{2}}{\sigma_{F}^{2}+\sigma_{T}^{2}} \beta \tilde{x}$ : this assumption, in conjunction with the $1^{\text {st }}$-order conditions, yields $P_{T}=\mu$, and when $P_{T}=\mu$ there is no additional information to be inferred from $P_{T}$. Below we discuss how our analysis extends to the case where $P_{T}$ is an additional source of information about $\tilde{\varepsilon}$ : see "Extension to $P_{T}$ as additional information."

Let $\Theta=\frac{\sigma_{F}^{2} \sigma_{T}^{2}}{\sigma_{F}^{2}+\sigma_{T}^{2}} \beta^{2}$. To determine $P_{a}$ when $P_{T}=\mu$, we employ the $1^{\text {st }}$-order conditions to derive investors' demands for the risky asset. This yields:

$$
D_{I a}=\tau \frac{E[\tilde{\varepsilon} \mid y]-\theta+x \lambda}{2 \tau \alpha N \lambda+\Theta+\operatorname{Var}[\tilde{\varepsilon} \mid y]} \text { and } D_{U a}=\tau \frac{\mu_{a}+E[\tilde{\varepsilon} \mid q]-P_{a}}{\Theta+\operatorname{Var}[\tilde{\varepsilon} \mid q]} .
$$

Using the same logic as in Section 3, one can use the demands for the risky asset in eqn. 
(37) to show

$$
\begin{aligned}
P_{a}-\mu_{a} & =\frac{\Theta+\operatorname{Var}[\tilde{\varepsilon} \mid q]}{\tau(1-\alpha) N}\left(\alpha N D_{I a}-x\right)+\frac{\pi_{\delta}}{\pi_{\varepsilon}+\pi_{\delta}}(\tilde{q}-E[\tilde{q}]) \\
& =\left(\frac{\Theta+\operatorname{Var}[\tilde{\varepsilon} \mid q]}{\tau(1-\alpha) N}+\frac{2 \lambda+\frac{1}{\tau \alpha N}(\Theta+\operatorname{Var}[\tilde{\varepsilon} \mid y])}{\frac{\pi_{\varepsilon}+\pi_{\delta}}{\pi_{\delta}} \frac{\pi_{\xi}}{\pi_{\varepsilon}+\pi_{\xi}}}\right)\left(\alpha N D_{I a}-x\right)-\frac{\pi_{\delta}}{\pi_{\varepsilon}+\pi_{\delta}} E[\tilde{q}],
\end{aligned}
$$

where here

$$
\begin{aligned}
\tilde{q} & =\tilde{y}-\frac{\pi_{\varepsilon}+\pi_{\xi}}{\pi_{\xi}}\left(\theta+\left(\lambda+\frac{1}{\tau \alpha N}(\Theta+\operatorname{Var}[\tilde{\varepsilon} \mid y])\right) \tilde{x}\right) \text { and } \\
\pi_{\delta} & =\left(\pi_{\xi}^{-1}+\left(\frac{\pi_{\varepsilon}+\pi_{\xi}}{\pi_{\xi}}\left(\lambda+\frac{1}{\tau \alpha N}(\Theta+\operatorname{Var}[\tilde{\varepsilon} \mid y])\right)\right)^{2} \pi_{x}^{-1}\right)^{-1} .
\end{aligned}
$$

To sustain the conjecture $P_{a}=\mu_{a}+\theta+\lambda\left(\alpha N D_{I a}-x\right)$, it must be the case that $\lambda$ solves

$$
\lambda=\frac{\Theta+\operatorname{Var}[\tilde{\varepsilon} \mid q]}{\tau(1-\alpha) N}+\frac{\pi_{\delta}\left(\pi_{\varepsilon}+\pi_{\xi}\right)}{\pi_{\xi}\left(\pi_{\varepsilon}+\pi_{\delta}\right)}\left(2 \lambda+\frac{1}{\tau \alpha N}(\Theta+\operatorname{Var}[\tilde{\varepsilon} \mid y])\right)
$$

and $\theta$ solves

$$
\theta=\frac{\pi_{\delta}}{\pi_{\varepsilon}} \frac{\pi_{\xi}+\pi_{\varepsilon}}{\pi_{\xi}-\pi_{\delta}}\left(\lambda+\frac{1}{\tau \alpha N}(\Theta+\operatorname{Var}[\tilde{\varepsilon} \mid y])\right)
$$

Our next step is to prove the following lemma.

Lemma 3. There exists a unique, positive $\lambda^{*}$ that satisfies eqn. (40). In turn, the $\lambda^{*}$ can be used to solve for a (unique) $\pi_{\delta}$ in eqn. (39) and (unique) $\theta$ in eqn. (41).

Proof to Lemma 3. Let $\pi_{T}$ represent the reciprocal of $\sigma_{T}^{2}$. Expressed in terms of precisions, $\Theta=\frac{\beta^{2}}{\pi_{F}+\pi_{T}}$. Define $\Lambda(\lambda, \cdot)$ as

$$
\Lambda(\lambda, \cdot)=\lambda-\frac{\Theta+\left(\pi_{\varepsilon}+\pi_{\delta}\right)^{-1}}{\tau(1-\alpha) N}-\frac{\pi_{\delta}\left(\pi_{\varepsilon}+\pi_{\xi}\right)}{\pi_{\xi}\left(\pi_{\varepsilon}+\pi_{\delta}\right)}\left(2 \lambda+\frac{1}{\tau \alpha N}\left(\Theta+\left(\pi_{\varepsilon}+\pi_{\xi}\right)^{-1}\right)\right)
$$

and then substitute in the expression for $\pi_{\delta}$ in eqn. (39) and then solve for $\lambda$. Ignoring 
proportionality factors, this yields the following expression for $\Lambda(\lambda, \cdot): \Lambda(\lambda, \cdot)=c_{3} \lambda^{3}+$ $c_{2} \lambda^{2}-c_{1} \lambda-c_{0}$, where the coefficients $c_{j}$ are defined by

$$
\begin{aligned}
c_{3}= & \left(N \tau\left(\pi_{F}+\pi_{T}\right)\right)^{3} \pi_{\varepsilon}\left(\pi_{\varepsilon}+\pi_{\xi}\right)^{2} \alpha^{2}(1-\alpha), \\
c_{2}= & \left(N \tau\left(\pi_{F}+\pi_{T}\right)\right)^{2}\left(\pi_{\varepsilon}+\pi_{\xi}\right) \alpha\left(\pi_{\varepsilon}(2-3 \alpha)\left(\pi_{F}+\pi_{T}+\beta^{2}\left(\pi_{\varepsilon}+\pi_{\xi}\right)\right)-\alpha\left(\pi_{F}+\pi_{T}\right) \pi_{\xi}\right), \\
c_{1}= & N \tau\left(\pi_{F}+\pi_{T}\right)\left(\left(N \tau \alpha\left(\pi_{F}+\pi_{T}\right)\right)^{2} \pi_{\xi}\left(\pi_{\varepsilon}+\pi_{\xi}\right) \pi_{x}(1-\alpha)\right. \\
& \left.+\left(\pi_{F}+\pi_{T}+\beta^{2}\left(\pi_{\varepsilon}+\pi_{\xi}\right)\right)\left(\pi_{\varepsilon}(3 \alpha-1)\left(\pi_{F}+\pi_{T}+\beta^{2}\left(\pi_{\varepsilon}+\pi_{\xi}\right)\right)+2 \alpha\left(\pi_{F}+\pi_{T}\right) \pi_{\xi}\right)\right), \\
c_{0}= & \left(\pi_{F}+\pi_{T}+\beta^{2}\left(\pi_{\varepsilon}+\pi_{\xi}\right)\right) \\
& \times\left(\left(\pi_{F}+\pi_{T}+\beta^{2} \pi_{\varepsilon}\right)\left(\pi_{F}+\pi_{T}+\beta^{2}\left(\pi_{\varepsilon}+\pi_{\xi}\right)\right)+\left(N \tau\left(\pi_{F}+\pi_{T}\right)\right)^{2} \alpha \pi_{\xi} \pi_{x}\right) .
\end{aligned}
$$

Note that $c_{3}$ and $c_{0}$ are both positive, but the signs of $c_{2}$ and $c_{1}$ are indeterminate. Because $c_{3}$ and $c_{0}$ are both positive, there exists some $\lambda>0$ that solves $\Lambda(\lambda, \cdot)=0$. For $\Lambda(\lambda, \cdot)=0$ to have multiple, positive $\lambda$-roots, Descartes' rule of signs requires that $c_{2}$ and $c_{1}$ both be negative (when $c_{3}$ and $c_{0}$ are both positive). A necessary and sufficient condition that $c_{2}$ is negative is that $\alpha>\frac{2 \pi_{\varepsilon}\left(\pi_{F}+\pi_{T}+\beta^{2}\left(\pi_{\varepsilon}+\pi_{\xi}\right)\right)}{3 \pi_{\varepsilon}\left(\pi_{F}+\pi_{T}+\beta^{2}\left(\pi_{\varepsilon}+\pi_{\xi}\right)\right)+\left(\pi_{F}+\pi_{T}\right) \pi_{\xi}}$, and a necessary (but not sufficient) condition that $c_{1}$ is negative is that $\alpha<\frac{\pi_{\varepsilon}\left(\pi_{F}+\pi_{\varepsilon}+\pi_{\xi}\right)}{3 \pi_{\varepsilon}\left(\pi_{F}+\pi_{T}+\beta^{2}\left(\pi_{\varepsilon}+\pi_{\xi}\right)\right)+2\left(\pi_{F}+\pi_{T}\right) \pi_{\xi}}$. But there is no $\alpha$ that satisfies both necessary conditions, and so $c_{2}$ and $c_{1}$ cannot both be negative: this establishes the existence of a unique $\lambda>0$ that satisfies $\Lambda(\lambda, \cdot)=0$. One can employ a proof nearly identical to the one in Proposition 1 to establish that $\frac{d}{d \lambda} \Lambda\left(\lambda^{*}, \cdot\right)>0$, and $\Lambda(\hat{\lambda}, \cdot)<0$ implies $\hat{\lambda}<\lambda^{*}$. Q.E.D.

Finally, and as in Section 3, we evaluate whether $\pi_{\varepsilon}$ and $\pi_{\xi}$ manifest in the risky asset's risk premium as the number of investors becomes large, where the risk premium is the difference in its expected cash flow relative to its beginning-of-period price: $\Delta(\lambda, \cdot)=E\left[\tilde{V}_{a}-\tilde{P}_{a}\right]$. Specifically, we prove the following proposition.

Proof to Proposition 4. First, determine $\Lambda(\lambda, \cdot)$ when $\pi_{F}^{-1}=N \pi_{f}^{-1}$ as $N$ becomes large. 
To do this, substitute in the expression for $\pi_{\delta}$ in eqn. (39) into eqn. (42) and then solve for $\lambda$ as $N$ becomes large; unlike the expression for $\Lambda(\lambda, \cdot)$ in Lemma 3, however, here do not ignore proportionality factors because these factors are a function of $N$. When it is either the case that there is no structural uncertainty (i.e., $\pi_{T} \rightarrow \infty$ ) or the uncertainty is finite (i.e., $\pi_{T}^{-1}$ is finite), the (unique, positive) $\lambda^{*}$ that solves $\Lambda\left(\lambda^{*}, \cdot\right)=0$ is $\lambda^{*}=\sqrt{\frac{\pi_{\xi} \pi_{x}}{\pi_{\varepsilon}\left(\pi_{\varepsilon}+\pi_{\xi}\right)}}$. Finally, determine $\Delta(\lambda, \cdot)$ when $\pi_{F}^{-1}=N \pi_{f}^{-1}$ as $N$ becomes large. Substituting in $\lambda^{*}=\sqrt{\frac{\pi_{\xi} \pi_{x}}{\pi_{\varepsilon}\left(\pi_{\varepsilon}+\pi_{\xi}\right)}}$ into $\Delta(\lambda, \cdot)$ yields $\Delta\left(\lambda^{*}, \cdot\right)=0$. To prove the second part of the proposition, now allow $\pi_{T}^{-1}=N \pi_{\mu}^{-1}$ and re-do the first part. Here $\Delta(\lambda, \cdot)$ does not go to 0 and has a unique, positive $\lambda$-root: call this root $\hat{\lambda}$. Next, show that $\Lambda(\hat{\lambda}, \cdot)<0$ and hence $\hat{\lambda}<\lambda^{*}$ (the proof is nearly identical to the one in Proposition 1 and so we simply assert this result in the Proof to Lemma 3$)$; this, in turn, implies that $\Delta\left(\lambda^{*}, \cdot\right)>0$ because $\Delta(\lambda, \cdot)$ increases as $\lambda$ increases. Finally, the expression for $\Delta\left(\lambda^{*}, \cdot\right)$ is

$$
\Delta\left(\lambda^{*}, \cdot\right)=\frac{\left(\alpha \lambda^{*}+\frac{\beta^{2}}{\tau\left(\pi_{f}+\pi_{\mu}\right)}\right) \lambda^{*}-\frac{\alpha \pi_{\xi} \pi_{x}}{\pi_{\varepsilon}\left(\pi_{\varepsilon}+\pi_{\xi}\right)}}{2 \alpha \lambda^{*}+\frac{\beta^{2}}{\tau\left(\pi_{f}+\pi_{\mu}\right)}}
$$

this expression is directly a function of $\pi_{\varepsilon}$ and $\pi_{\xi}$, and indirectly a function of $\pi_{\varepsilon}$ and $\pi_{\xi}$ through $\lambda^{*}$. Q.E.D.

Extension to $P_{T}$ as additional information. The extension to the general case where $P_{T}$ provides additional information is straightforward in that it requires primarily that one re-define $\pi_{\delta}$. To elaborate on this issue, start by letting $\tilde{z}$ represent the random supply of the common factor available for trade, where $\tilde{z}$ has a normal distribution with mean 1 and precision $\pi_{z}$. Using the $1^{\text {st }}$-order conditions, the price of the common factor reduces to

$$
P_{T}=\mu-\frac{1}{N \tau \pi_{F}}\left(\frac{\pi_{F}+\pi_{T}}{\pi_{T}} \tilde{z}+\beta \tilde{x}\right) .
$$


Because $P_{T}$ depends on $\tilde{x}$, an uninformed investor can use $P_{T}$ to infer information about $\tilde{x}$ with "noise" (i.e., $\tilde{z}$ ), which, in turn, can be used to improve his inference about $\tilde{y}$ from $P_{a}$. For example, define the statistic $\tilde{r}$ by

$$
\tilde{r}=\frac{\mu-P_{T}}{\frac{1}{N \tau \pi_{F}} \beta}=\tilde{x}+\frac{\pi_{F}+\pi_{T}}{\beta \pi_{T}} \tilde{z}
$$

Note that $\tilde{r}$ has a normal distribution with a mean of $E[\tilde{r}]=1+\frac{\pi_{F}+\pi_{T}}{\beta \pi_{T}}$ and variance $\operatorname{Var}[\tilde{r}]=\pi_{x}^{-1}+\left(\frac{\pi_{F}+\pi_{T}}{\beta \pi_{T}}\right)^{2} \pi_{z}^{-1}$. Let $\boldsymbol{\Pi}$ represent the 1 -by-2 vector $\boldsymbol{\Pi}=\left(\pi_{\varepsilon}^{-1}, 0\right)$ and $\mathbf{Q}$ the 2-by-2 matrix

$$
\mathbf{Q}=\left(\begin{array}{cc}
\operatorname{Var}[\tilde{q}] & \operatorname{Cov}[\tilde{q}, \tilde{r}] \\
\operatorname{Cov}[\tilde{q}, \tilde{r}] & \operatorname{Var}[\tilde{r}]
\end{array}\right)
$$

where $\tilde{q}$ is defined as in eqn. (38). By conditioning their beliefs on $P_{a}$ and $P_{T}$, uninformed investors will assess the mean and variance of $\tilde{\varepsilon}$ conditional on $\tilde{q}=q$ and $\tilde{r}=r$ as:

$$
E[\tilde{\varepsilon} \mid q, r]=\boldsymbol{\Pi} \cdot \mathbf{Q}^{-1} \cdot\left(\begin{array}{c}
q-E[\tilde{q}] \\
r-E[\tilde{r}]
\end{array}\right) \text { and } \operatorname{Var}[\tilde{\varepsilon} \mid q, r]=\pi_{\varepsilon}^{-1}-\mathbf{\Pi} \cdot \mathbf{Q}^{-1} \cdot \mathbf{\Pi}^{T} .
$$

Having defined $E[\tilde{\varepsilon} \mid q, r]$ and $\operatorname{Var}[\tilde{\varepsilon} \mid q, r]$, the expressions for $\lambda, \theta$, and $\Delta(\lambda, \cdot)$ remain roughly similar (but not identical) to eqns. (40), (41), and (42). In effect, one can extend Proposition 4 to the case where uninformed investors can use $P_{T}$ to infer information about $\tilde{x}$. The extension is very complicated, however, and yields little additional insight. 